\title{
Photocrosslinkable Star Polymers via RAFT-Copolymerizations with $N$-Ethylacrylate-3,4-dimethylmaleimide
}

\author{
Nadja Förster ${ }^{1}$, Ann-Christin Pöppler ${ }^{2}$, Dietmar Stalke ${ }^{2}$ and Philipp Vana ${ }^{1, *}$ \\ 1 Institute of Physical Chemistry, Georg-August-University Göttingen, Tammannstr. 6, \\ D-37077 Göttingen, Germany; E-Mail: nadjafoerster@yahoo.de \\ 2 Institute of Inorganic Chemistry, Georg-August-University Göttingen, Tammannstr. 4, \\ D-37077 Göttingen, Germany; E-Mails: apoeppler@chemie.uni-goettingen.de (A.-C.P.); \\ dstalke@chemie.uni-goettingen.de (D.S.) \\ * Author to whom correspondence should be addressed; E-Mail: pvana@uni-goettingen.de; \\ Tel.: +49-551-39-33140; Fax: +49-551-39-12709.
}

Received: 25 April 2013; in revised form: 30 May 2013 / Accepted: 30 May 2013 /

Published: 10 June 2013

Abstract: This paper describes the Z-RAFT-star copolymerization of $n$-butyl acrylate (BA) and $N$-isopropyl acrylamide (NIPAm), respectively, with $\mathrm{N}$-ethylacrylate-3,4-dimethylmaleimide (1.1), a monomer carrying a UV-reactive unit that undergoes photocrosslinking. Addition of $\mathbf{1 . 1}$ slows down the polymerization rate both for BA and for NIPAm polymerization. Double star formation due to radical attack to the 3,4-dimethylmaleimide moiety was found in the case of BA. Dead polymer formation, presumably due to aminolysis as side-reaction, was pronounced in the NIPAm system. These two effects broadened the molar mass distributions, but did not impede the formation of functional star polymers. The composition of the copolymers as well as the reactivity ratios for the applied comonomers were determined via NMR spectroscopy $\left(\mathrm{BA}-c o-1.1 r_{1.1}=2.24 r_{\mathrm{BA}}=0.95\right.$; NIPAm-co-1.1 $\left.r_{\mathbf{1 . 1}}=0.96 r_{\mathrm{NIPAm}}=0.05\right)$. In both cases, the comonomer is consumed preferably in the beginning of the polymerization, thus forming gradient copolymer stars with the UV-reactive units being located in the outer sphere.

Keywords: RAFT polymerization; $N$-ethylacrylate-3,4-dimethylmaleimide; star polymers; $\mathrm{N}$-isopropyl acrylamide (NIPAm); butyl acrylate (BA); copolymerization; reactivity ratios 


\section{Introduction}

The internal crosslinking of a polymeric entity (single chain or molecular ensemble) is a powerful way to stabilize its shape in solution and thus, to achieve a stable functional particle. Following this approach, amphiphilic nano-carriers for drug-delivery can be generated by crosslinking of star-shaped polymers from RAFT-polymerization [1-5], as we are reporting elsewhere [6]. The aimed nano-carriers are established by using multifunctional, star-shaped RAFT-agents in block copolymerizations with monomers carrying a crosslinkable unit. Thereby, star block copolymers with outer blocks that may undergo internal crosslinking are available. There are different reaction mechanisms for such crosslinking reactions, which can be used to conduct the formation of a carrier's shell: On the one hand, functional groups introduced into the polymer by appropriate monomers can be linked via additional crosslinkers. Known examples are esterification reactions between hydroxyl-groups being part of the polymer and bifunctional acid chlorides or dichlorosilane compounds [7], de-epoxidation of glycidyl methacrylate (GMA) units with the bifunctional cystamine [8], as well as the 1,3-dipolar Huisgen-cycloaddition (so called "click-reaction") between alkynes and azide-groups [9,10]. In all of these cases, it is essential to obey an exact stoichiometry, which is difficult as there are usually uncertainties in the determination of the polymer's molar mass-commonly determined via Size-Exclusion Chromatography (SEC) — and uncertainties in the content of linkable comonomer - e.g., determined via NMR spectroscopy. For these types of crosslinking reactions, the systems also have to be rather dry and clean to assure complete conversion, which is essential for effective crosslinking. On the other hand, crosslinking between monomer units themselves is plausible, for which they need to carry dedicated functional moieties. In this case, the coupling reaction takes place without additional compounds: The reaction is triggered by favorable conditions, e.g., change in temperature or irradiation with light of appropriate wavelength, or bifunctional monomers, such as ethylene glycol dimethylacrylate (EGDMA) [11] or divinylbenzene [12], can be used. Another example is the reaction of cyclobutene at high temperatures $\left(T \approx 250{ }^{\circ} \mathrm{C}\right)$ of which, two form eight-membered rings [13]. In this kind of crosslinking procedure, the danger of polymer decomposition by heat is given. A non-destructive and versatile pathway is provided by photocrosslinking via [2+2]-cycloaddition stimulated by UV-irradiation, since wavelength and applied energy entry can be easily controlled. Many photocrosslinkable monomers can be found in the literature, e.g., based on $N$-alkyl-3,4-dimethylmaleimid (DMI) [14], anthracene [15,16], stilbene [17], cinnamic acid [18,19], uracil derivatives [20] or coumarine [21].

This paper presents the results of the RAFT-mediated copolymerizations of $n$-butyl acrylate (BA) and $N$-isopropyl acrylamide (NIPAm) with the UV-reactive monomer $N$-ethylacrylate-3,4-dimethylmaleimide (1.1, Figure 1) mediated by six-functional star-shaped RAFT-agents that follow the so-called Z-group approach (Figure 2), where RAFT moieties are linked to a central core via the stabilizing Z-group. The beneficial consequence of this strategy is that (i) radical centers never sit on the star polymer, which reduces star-star coupling via termination, and that (ii) no linear controlled polymer is formed next to the stars, as the RAFT groups never depart from the star molecules. For details about Z-RAFT star polymerization see [22]. 
Figure 1. The monomers $n$-butyl acrylate (BA), $N$-isopropyl acrylamide (NIPAm) and $\mathrm{N}$-ethylacrylate-3,4-dimethylmaleimide (1.1).
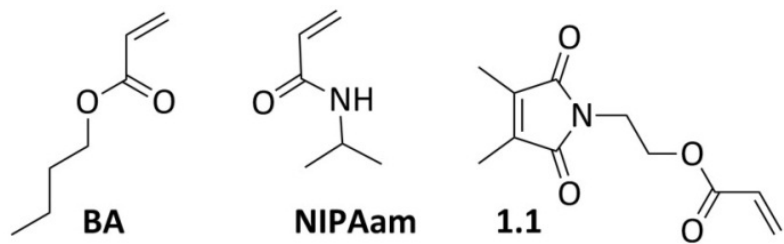

Figure 2. The RAFT agents dipentaerythriol-hexakis(3-S-phenylethyl-tricarbonylpropanoate) $\mathbf{R}_{\mathbf{6}} \mathbf{2}$ for BA and dipentaerythriol-hexakis(3-S-(methyl-2-propanoatotrithiocarbonyl)propanoate) $\mathbf{R}_{\mathbf{6}} \mathbf{3}$ for NIPAAm polymerization [22-24].<smiles>[R][R]OC(=O)CCSC(=S)S[R][R]</smiles>

In order to place the functional monomers more or less evenly distributed along the polymer backbone - which is beneficial for homogeneous crosslinking - the respective reactivity ratios of the two involved comonomers, i.e., the basic monomer and the monomer carrying the UV-reactive unit, should both be close to one. It was expected in this work that the $\mathrm{C}_{2}$-bridge in $\mathbf{1 . 1}$ is sufficient to separate the reactive double bond for polymerization from the UV-reactive rest of the monomer, i.e., the polymerization activity of $\mathbf{1 . 1}$ was assumed to be close to the one of butyl acrylate (BA). NIPAm was also tested in copolymerization with $\mathbf{1 . 1}$ in order to obtain photocrosslinkable hydrophilic polymer. The dissimilarity of $\mathbf{1 . 1}$ and NIPAm (acrylate $v s$. acrylamide), however, may lead to deviations from reactivity ratios being close to one. An inhomogeneous distribution of functional groups along the polymer backbone may consequently result, as the chain growth is controlled by RAFT throughout the polymerization, and gradient copolymers may thus be formed. The present report considers these aspects, and is the first one that studies the copolymerization of $\mathbf{1 . 1}$ in RAFT-polymerizations, as well as providing information that is necessary to obtain functional polymers that may undergo homogeneous and reproducible photocrosslinking. 


\section{Experimental Section}

\subsection{Materials}

Butyl acrylate (BA, Aldrich, $\geq 99 \%$ ) was freed from inhibitor by passing through a column of neutral aluminium oxide (Aldrich, Brockmann activity I). $N$-isopropyl acrylamide (NIPAm, Acros Organics, 99\%) was re-crystallized from $n$-hexane. Azo-bis-(isobutyronitrile) (AIBN, AkzoNobel, 98\%) was re-crystallized from methanol. Solvents used in syntheses and polymerizations were purchased from different suppliers in pro analysi quality and used as received. Tetrahydrofuran (THF) and $N, N$-dimethylacetamide (DMAc) for SEC-analysis were purchased in HPLC-grade from Sigma-Aldrich. NMR-solvents were purchased from Deutero or Aldrich (99.8 atom \% D) and used as received.

\subsection{Syntheses}

The comonomer $N$-ethylacrylate-3,4-dimethylmaleimide $\mathbf{1 . 1}$ was synthesized in accordance to literature-known procedures: First, the precursor dimethylmaleimido ethanol was synthesized in accordance to Gupta et al. in 98\% yield [25]. Afterwards, it was used without further purification in a Steglich esterification with acrylic acid (overall yield 80\%) [26].

The six-functional RAFT-agents $\mathbf{R}_{\mathbf{6}} \mathbf{2}$ and $\mathbf{R}_{\mathbf{6}} \mathbf{3}$ were synthesized as described earlier [22-24].

\subsection{Polymerizations}

Solvent and liquid monomers were degassed via at least three freeze-pump-thaw cycles. Along with the polymerization vials, the RAFT-agent, initiator, and the comonomer were transferred to an argon-filled glove box (oxygen-content below $2 \mathrm{ppm}$ ). There, the stock solutions for the polymerization were prepared and charged to glass vials and sealed with Teflon/rubber lids. The vials were inserted into a block heater at $60 \pm 0.1^{\circ} \mathrm{C}$ and removed after preset time intervals. After cooling in an ice bath for quenching the reaction, the monomer-to-polymer conversion was determined gravimetrically by precipitating the polymer from the reaction mixture (poly-BA with methanol, poly-NIPAm with diethyl ether). The polymer was separated via centrifugation and dried under vacuum.

\subsection{Size Exclusion Chromatography (SEC)}

The molecular weight distributions of BA-based polymers were determined with a SEC Analysis Systems 1260 Infinity by PSS Agilent (PSS Agilent Technologies 1260 Iso Pump, Agilent 1260 ALS injector, pre-column PSS SDV, $8 \mathrm{~mm} \times 50 \mathrm{~mm}$, particle size: $5 \mu \mathrm{m}$, three separation columns PSS SDV, $8 \mathrm{~mm} \times 300 \mathrm{~mm}$, particle size: $5 \mu \mathrm{m}$, pore size: $10^{5}, 10^{3}$ and $10^{2} \AA$ ) with the UV-detector PSS Agilent Technologies 1260 VWDVL at a wavelength of $310 \mathrm{~nm}$ [27] and the RI-detector PSS Agilent Technologies 1260 RID. As eluent THF was used with toluene ( $>99.7 \%$, dry, from Sigma-Aldrich) as internal standard (flow velocity $1.0 \mathrm{~mL} \cdot \mathrm{min}^{-1}$ at $35{ }^{\circ} \mathrm{C}$ ). The system was calibrated with polystyrene standards of low dispersity from PSS. The Mark-Houwink-coefficients (BA: $K=0.0122 \mathrm{~cm}^{3} \cdot \mathrm{g}^{-1}$, $a=0.7$; Sty: $K=0.0162 \mathrm{~cm}^{3} \cdot \mathrm{g}^{-1}, a=0.71$ ) [28,29] were used to gain access to the molecular masses of the homopolymers according to the principles of universal calibration. The molecular weight 
distributions of poly-NIPAm samples were determined with a SEC Analysis Systems 1260 Infinity set up by PSS Agilent containing a PSS GRAM (polyester copolymer network) pre-column, $8 \mathrm{~mm} \times 50 \mathrm{~mm}$ and three PSS GRAM separation columns, $8 \mathrm{~mm} \times 300 \mathrm{~mm}$, particle size: $10 \mu \mathrm{m}$, pore sizes: $30,10^{3}$ and $10^{3} \AA$. As eluent DMAc with $0.1 \mathrm{wt} \% \mathrm{LiBr}$ was used (flow velocity $0.8 \mathrm{~mL} \cdot \mathrm{min}^{-1}$ at $45^{\circ} \mathrm{C}$ ). The UV-detector PSS Agilent Technologies $1260 \mathrm{VWDVL}$ at a wavelength of $310 \mathrm{~nm}$ [27] and the RI-detector PSS Agilent Technologies 1260 RID were applied. Again toluene was used as internal standard. The system was calibrated with poly-MMA standards from PSS. McKee et al. proved by using NMR-analysis parallel to the SEC measurements that poly-NIPAm behaves very similar to poly-MMA in solution [30].

\subsection{NMR Spectroscopy}

Different NMR-experiments $\left({ }^{1} \mathrm{H}\right.$ NMR, $\left.{ }^{15} \mathrm{~N}-\mathrm{HMBC}, \mathrm{H}, \mathrm{H}-\mathrm{COSY}\right)$ were conducted on a Bruker Avance III $400 \mathrm{MHz}$ spectrometer (BrukerBiospin, Rheinstetten) with a broadband-observe probe, $z$-gradient and temperature unit. All measurements were performed in $\mathrm{CDCl}_{3}$ applying a relaxation time $d_{1}$ of $0.1 \mathrm{~s}$ and a pulse length $p_{1}$ of $10 \mu \mathrm{s}$. As no literature data was available for the ${ }^{15} \mathrm{~N}$ chemical shift of the comonomer $\mathbf{1 . 1}$ it had to be characterized thoroughly. Acetonitrile was applied as nitrogen standard. Furthermore, the chemical shifts of the ethyl-bridge protons in the ${ }^{1} \mathrm{H}$ NMR spectrum had to be clarified. A two-dimensional NMR method, ${ }^{15} \mathrm{~N}-\mathrm{HMBC}$ (Heteronuclear Multiple Bond Correlation), was applied to yield straightforward data despite the low natural abundance $(0.37 \%)$ of ${ }^{15} \mathrm{~N}$. In a ${ }^{15} \mathrm{~N}-\mathrm{HMBC}$ experiment the connectivity information along several bonds between the ${ }^{15} \mathrm{~N}$ and ${ }^{1} \mathrm{H}$ nuclei is detected.

\section{Results and Discussion}

\subsection{Copolymerization of 1.1 and $B A$}

Polymerizations of BA and $\mathbf{1 . 1}$ were conducted in a BA: toluene mixture of a volume ratio of 7:3, which corresponds to $c a .5000 \mathrm{mmol} \cdot \mathrm{L}^{-1}$ BA. $0.6 \mathrm{mmol} \cdot \mathrm{L}^{-1} \mathbf{R}_{\mathbf{6}} \mathbf{2}$ and $1.1 \mathrm{mmol} \cdot \mathrm{L}^{-1}$ AIBN were added and the reactions were carried out at $60{ }^{\circ} \mathrm{C}$ for up to $155 \mathrm{~min}$. The concentration of $\mathbf{1 . 1}$ was varied systematically between 29 and $279 \mathrm{mmol} \cdot \mathrm{L}^{-1}$. With these amounts of $\mathbf{1 . 1}$, the final crosslink density may roughly be varied between 1 crosslink per 200 monomer units and 1 crosslink per 20 monomer units. In Figure 3 the number average molecular weights, $M_{\mathrm{n}}$, and the dispersities, $\oslash$, of these different polymerizations are plotted against the monomer conversion.

A controlled process of the polymerization can be recorded for all copolymerizations in toluene, even for the higher comonomer concentrations, proven by the linear development of $M_{\mathrm{n}}$ with monomer conversion as illustrated in Figure 3 and by the SEC-traces (shown in the electronic supporting information (ESI) Figure S1). In general, it has to be noted that the $\oslash$-values of these solvent copolymerizations are higher than typically obtained in Z-RAFT star polymerizations, where values below 1.25 were obtained [22]. On the one hand this may be due to the good thermodynamic dissolution quality of the applied solvents, as could be shown for solvent Z-RAFT star polymerizations of styrene earlier [31]. On the other hand, it is observed that the $\oslash$-values are getting higher for higher concentrations of $\mathbf{1 . 1}$ in the monomer feed. The corresponding SEC-traces show bimodal distributions 
which cause the high $\oslash$-values (Figure 4 and Figure S1 in ESI). It is noticeable that the formation of material with higher molecular weight causing bimodality depends on the comonomer concentration and reaction time. Here the chromatograms obtained by RI-detection are shown. The molar mass distributions after long reaction times (Figure $4 \mathrm{~b}$ ) additionally show a shoulder at smaller molecular weights. These result from dead material, which is generated during the RAFT polymerization process due to continuous termination of two individual arm macroradicals, which naturally are smaller than the complete star consisting of six arms (main peak). As the low molar mass material is formed by termination of propagating radicals, it does not contain RAFT-units.

Figure 3. Developments of the number average molecular weight, $M_{\mathrm{n}}$, and dispersity, $\oslash$, with the monomer conversion for homopolymerization of BA and four copolymerizations of BA and 1.1 with $0.6 \mathrm{mmol} \cdot \mathrm{L}^{-1} \mathbf{R}_{\mathbf{6}} 2$ and $1.1 \mathrm{mmol} \cdot \mathrm{L}^{-1} \mathrm{AIBN}$ in toluene at $60{ }^{\circ} \mathrm{C}$.

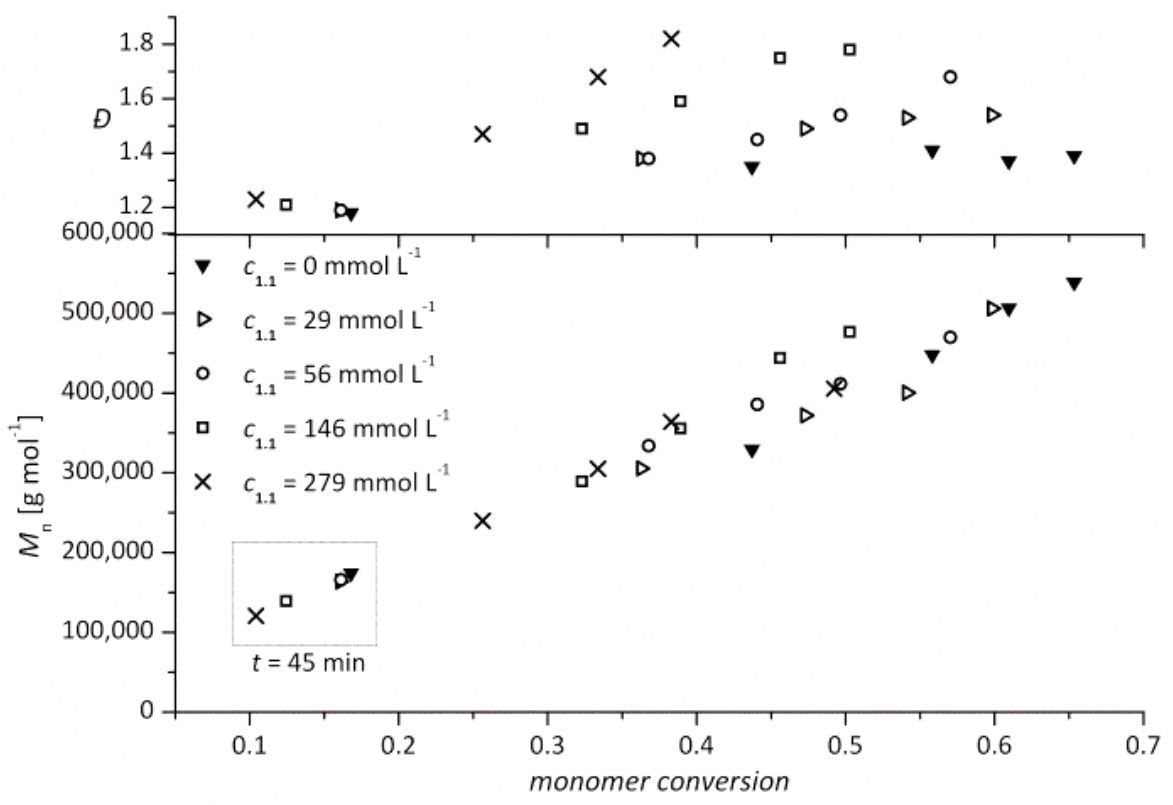

Figure 4. Molar mass distributions (SEC) from different copolymerizations of BA with $\mathbf{1 . 1}$ in toluene with $0.6 \mathrm{mmol} \cdot \mathrm{L}^{-1} \mathbf{R}_{\mathbf{6}} \mathbf{2}$ and $1.1 \mathrm{mmol} \cdot \mathrm{L}^{-1} \mathrm{AIBN}$ at $60{ }^{\circ} \mathrm{C}$ taken after $45 \mathrm{~min}(\mathbf{a})$, $155 \min (\mathbf{b})$.

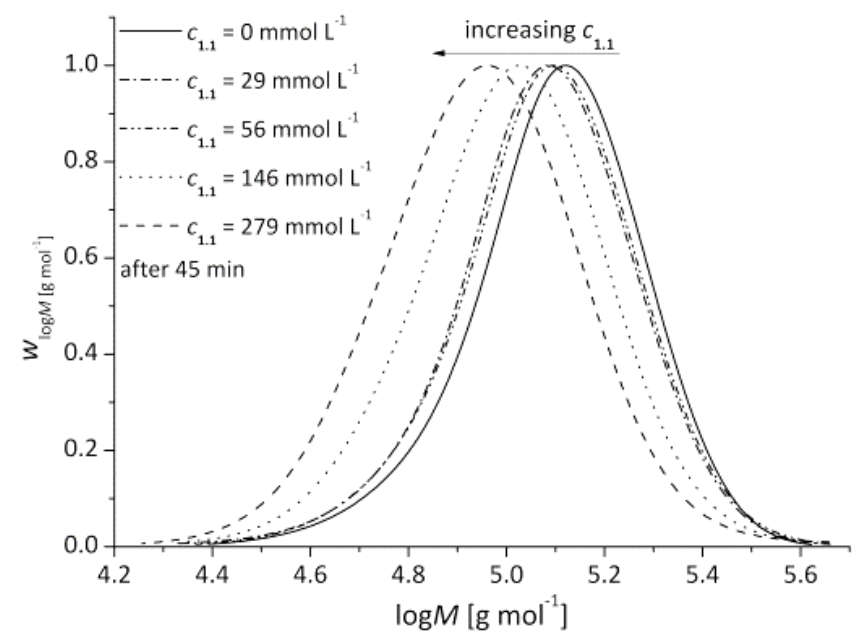

(a)

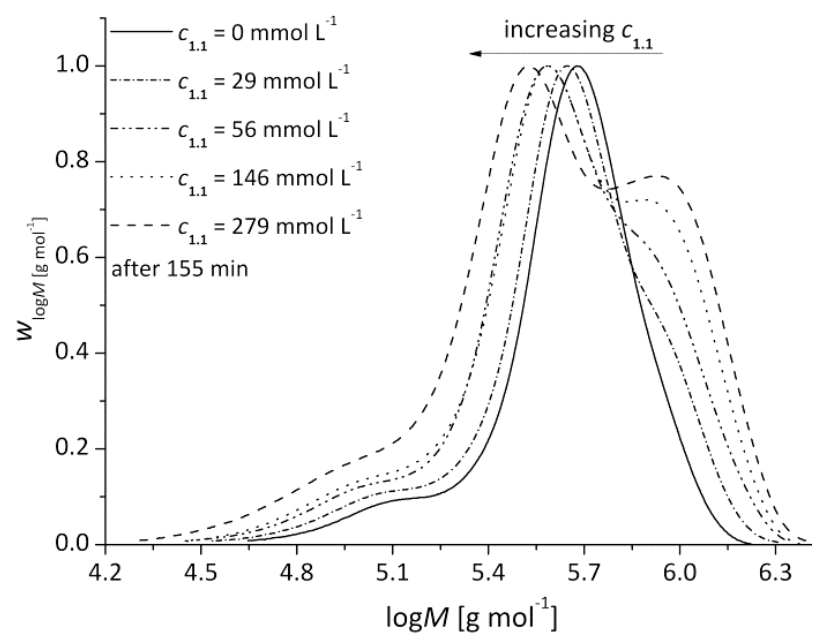

(b) 
With increasing concentration of the comonomer, decreasing molar masses are obtained after identical reaction times (see Figure 5, lower part), which directly reflects a slowing down in polymerization rate, whereas the formation of dead polymer proceeds approximately to the same extent for all cases. The plots of monomer conversion $v s$. reaction time (Figure 5, upper part) confirm these qualitative findings and indicate that increasing amounts of $\mathbf{1 . 1}$ as comonomer slow down the reaction and thus reduces molar masses after identical reaction periods. This rate reduction is often observed with copolymerizations and may here originate from the steric congestion when the bulky monomer 1.1 adds to the BA macroradical, which consequently slows down propagation due to a reduced frequency factor of the corresponding Arrhenius equation.

Figure 5. Developments of monomer conversion and number average molecular weight with time in homopolymerization of BA and in different copolymerizations of BA and $\mathbf{1 . 1}$ in toluene using $0.6 \mathrm{mmol} \cdot \mathrm{L}^{-1} \mathbf{R}_{\mathbf{6}} \mathbf{2}$ and $1.1 \mathrm{mmol} \cdot \mathrm{L}^{-1} \mathrm{AIBN}$ at $60^{\circ} \mathrm{C}$.

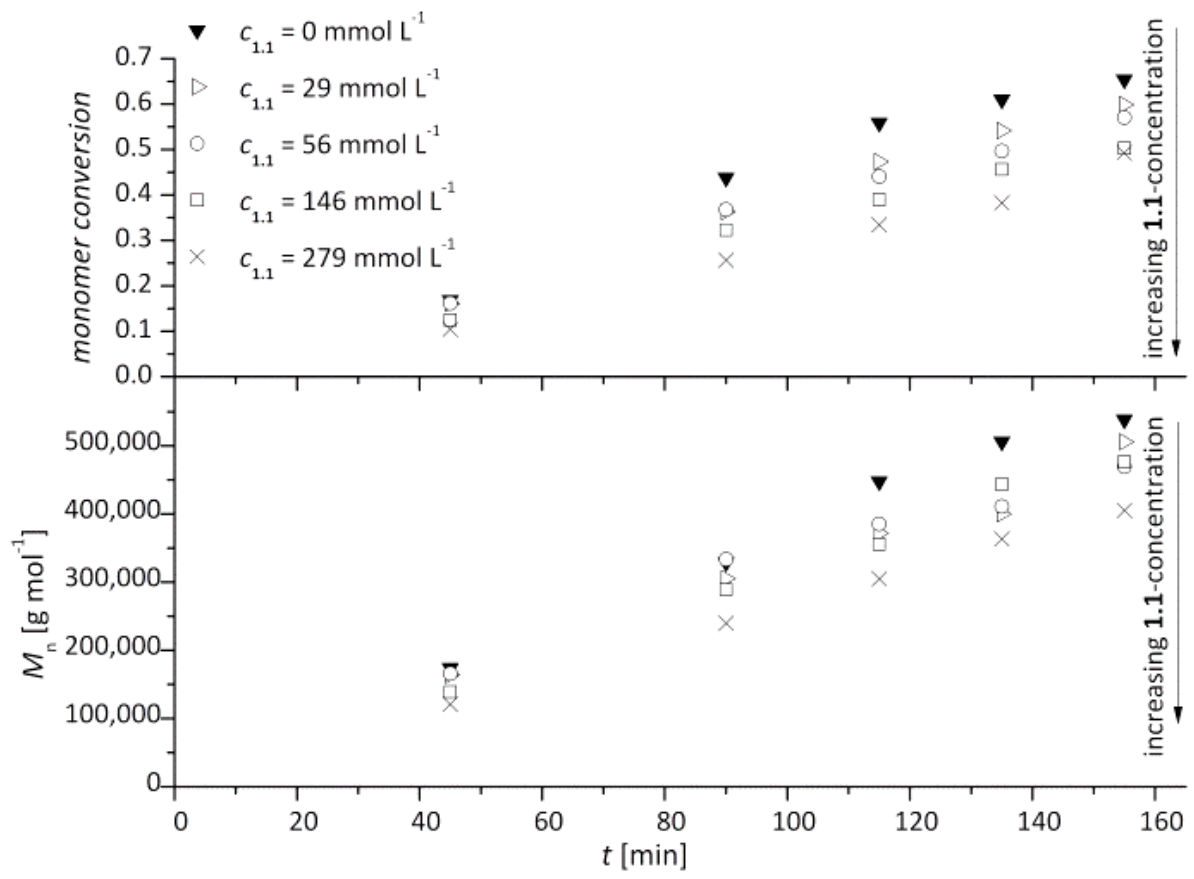

For higher monomer conversion, i.e., here after $155 \mathrm{~min}$, a shoulder in the region of high molecular weight can be seen besides the main signal which results from star polymer and dead material. We showed earlier that in Z-RAFT star polymerization of BA this high molar mass shoulder was caused by star-star coupling via intermolecular transfer-to-polymer [22]. By $\beta$-H-abstraction from the polymer backbone by a propagating radical, an intermolecular transfer of the radical functionality takes place. A tertiary radical, a so called mid-chain radical, is formed and dead polymer is generated. This midchain radical does not undergo RAFT-equilibrium reactions due to its hindered nature, but propagates, forming a secondary radical. This star radical species, which normally does not occur in Z-RAFT star polymerization, subsequently participates in the RAFT equilibrium yielding star couples. However, as the polymerization shown here was carried out in solution (monomer:solvent 7:3), the effect of star-star-coupling via transfer-to-polymer is expected to be small, due to the reduced polymer concentration. It is furthermore evident that in polymerizations with higher comonomer feed, the multimodality of the distribution is much more pronounced and that poly-BA homopolymer does not 
show this high molar mass fraction at all (see Figure 4). These findings imply that in this particular case the coupling originates via some other mechanism.

The examination of the chromatograms resulting from the 'corrected' UV-data (Figure 6), which selectively detects polymer carrying RAFT groups only, confirms an increase of the high molecular weight side product, both with increasing comonomer concentration and reaction time. The correction of the UV-data was conducted in accordance to the procedure described earlier [22] and accounts for the fact that the UV detector is set to a wavelength by which only RAFT groups are visible. The corresponding UV signal is proportional to the number of molecules instead of being proportional to the weight of the polymer, as is true for RI-detection. Correcting the UV-signal, i.e., multiplying by the molar mass, thus makes UV and RI signals comparable weight distributions, which should be identical in the case that every chain is carrying the same number of RAFT-groups. With star couples, however, two absorbing RAFT centers occur per macromolecule, which amplifies the corresponding corrected UV signal by a factor of two, giving evidence for the molecular structure of this star couple. This becomes clear from inspection of Figure 6, which directly proves that progressive star-star coupling occurs during the studied copolymerization.

Figure 6. Corrected UV-SEC-traces of the aliquots quenched after 115 (a) and $155 \mathrm{~min}$ (b) of copolymerizations of BA and different amounts of 1.1 using $0.6 \mathrm{mmol} \cdot \mathrm{L}^{-1} \mathbf{R}_{\mathbf{6}} \mathbf{2}$ and $1.1 \mathrm{mmol} \cdot \mathrm{L}^{-1} \mathrm{AIBN}$ at $60^{\circ} \mathrm{C}$, (for details see text).

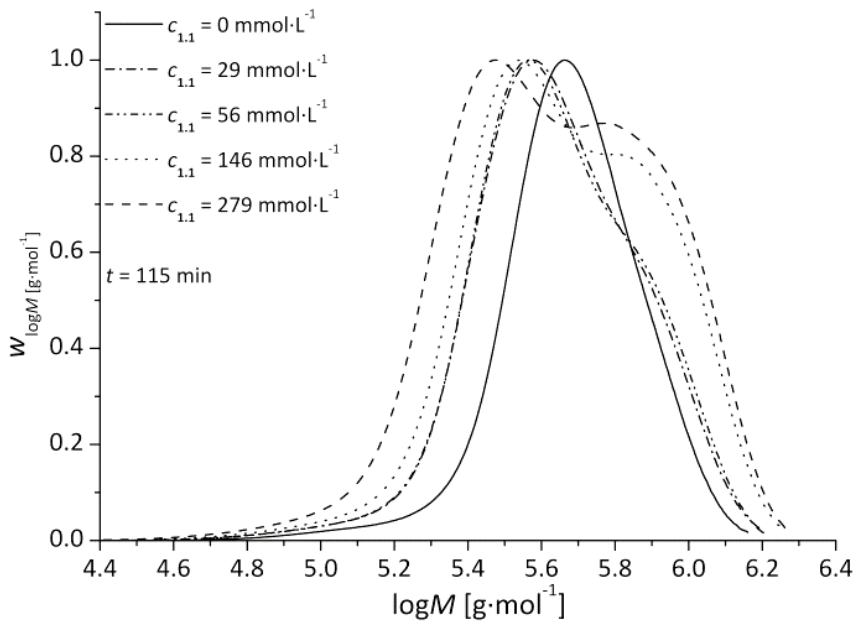

(a)

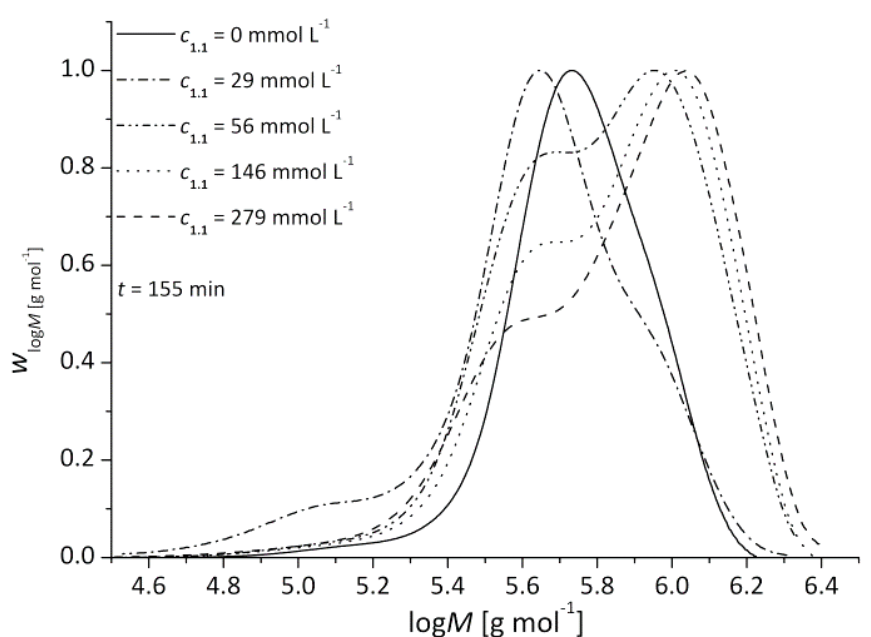

(b)

It can be concluded that the dimethylmaleimide-moiety is prone to undergo side reactions which yield polymer material with higher molecular weights than a single star polymer. The assumption seems plausible that the probability of star-star coupling is intensified by these units. Of the copolymerizations with 56,146 and $279 \mathrm{mmol} \cdot \mathrm{L}^{-1}$ of 1.1, each fourth and fifth aliquots were probed for star-star-coupling, which results in doubling of the molecular mass (Table 1). Here, the molecular weights of the peak maxima $\left(M_{\mathrm{P}}\right)$ of both peaks were compared. " $M_{\mathrm{P} \text {,high" }}$ " corresponds to the molecular weight at the peak maximum of the high molecular weight material, i.e., multi-stars, " $M_{\mathrm{P}, \text { low }}$ " to the peak maximum of the peak of the material with the smaller molecular weight, i.e., single stars. 
Table 1. Comparison of the molecular weights of the two maxima of the MWDs of selected aliquots resulting from the copolymerization of BA and different amounts of $\mathbf{1 . 1}$ using $0.6 \mathrm{mmol} \cdot \mathrm{L}^{-1} \mathbf{R}_{\mathbf{6}} \mathbf{2}$ and $1.1 \mathrm{mmol} \cdot \mathrm{L}^{-1} \mathrm{AIBN}$ at $60{ }^{\circ} \mathrm{C}$.

\begin{tabular}{|c|c|c|c|}
\hline Aliquot & $M_{\mathrm{P}, \text { high }}\left(\mathrm{g} \cdot \mathrm{mol}^{-1}\right)$ & $M_{\mathrm{P}, \text { low }}\left(\mathrm{g} \cdot \mathrm{mol}^{-1}\right)$ & Ratio \\
\hline $4\left(0.056 \mathrm{~mol} \cdot \mathrm{L}^{-1}\right)$ & 740,000 & 398,000 & 1.86 \\
\hline $5\left(0.056 \mathrm{~mol} \cdot \mathrm{L}^{-1}\right)$ & 890,000 & 490,000 & 1.82 \\
\hline $4\left(0.146 \mathrm{~mol} \cdot \mathrm{L}^{-1}\right)$ & 980,000 & 447,000 & 2.19 \\
\hline $5\left(0.146 \mathrm{~mol} \cdot \mathrm{L}^{-1}\right)$ & $1,000,000$ & 470,000 & 2.12 \\
\hline $4\left(0.279 \mathrm{~mol} \cdot \mathrm{L}^{-1}\right)$ & 933,000 & 355,000 & 2.63 \\
\hline $5\left(0.279 \mathrm{~mol} \cdot \mathrm{L}^{-1}\right)$ & $1,100,000$ & 407,000 & 2.69 \\
\hline
\end{tabular}

The assumption of double star formation is further strengthened by these data, as $M_{\mathrm{P}, \text { low }}$ is in all cases approximately about a factor of 2 smaller than $M_{\mathrm{P} \text {,high. }}$ Furthermore, a trend can be observed, as the ratio between the molecular weights is increasing with increasing comonomer concentration. For the highest comonomer concentration, the ratio is almost threefold larger, which indicates that entities consisting of more than two stars are formed to some extent. A thermal initiation of the $[2+2]$-cycloaddition is thermodynamically forbidden. The only conceivable pathway is therefore via the reaction of a radical with the dimethylmaleimide-moiety: Maleimides themselves are nominally 1,2-disubstituted ring-shaped vinyl monomers and may be attacked by a growing radical. Disubstituted double bonds are known to propagate very slowly [32]. Maleimide is nevertheless known to undergo copolymerizaton with styrene [33] and with acrylates [34] to some extent. Additional substitution of the double bond with two methyl groups on either side is further reducing the probability of being partner in a radical polymerization. It can thus safely be anticipated that propagation of $\mathbf{1 . 1}$ occurs via radical attack to the acrylic side of the monomer. However, a single event on the maleimide side, although being very unlikely, will result in the incorporation of this monomer into two polymeric chains, that is, in the formation of a star-star couple. It is thus believed that the star-star couples in Z-RAFT star copolymerization of BA and $\mathbf{1 . 1}$ originates from such sporadic macroradical attack to the dimethylmaleimide-moiety. The increasing amount of dimethylmaleimide units in the system by increasing the comonomer feed of $\mathbf{1 . 1}$ is thus directly increasing the amount of star-star couples.

\subsection{Copolymerization of 1.1 and NIPAm}

Copolymerizations of NIPAm and 1.1, mediated by the six-functional RAFT agent $\mathbf{R}_{\mathbf{6}} \mathbf{3}$, were also studied. Solutions of $5000 \mathrm{mmol} \cdot \mathrm{L}^{-1}$ of NIPAm and $28 \mathrm{mmol} \cdot \mathrm{L}^{-1}, 56 \mathrm{mmol} \cdot \mathrm{L}^{-1}, 140 \mathrm{mmol} \cdot \mathrm{L}^{-1}$ and $280 \mathrm{mmol} \cdot \mathrm{L}^{-1}$ of $\mathbf{1 . 1}$ in DMF were polymerized with $0.6 \mathrm{mmol} \cdot \mathrm{L}^{-1}$ of $\mathbf{R}_{\mathbf{6}} \mathbf{3}$ and $1.1 \mathrm{mmol} \cdot \mathrm{L}^{-1}$ of AIBN at $60{ }^{\circ} \mathrm{C}$. In Figure 7 the corresponding development of the molecular weight and the dispersities with monomer conversion are plotted.

It can be seen that the molecular weights increase in a linear fashion with monomer conversion and that the $\oslash$-values for polymerizations with no or a low amounts of comonomer in the monomer feed are reasonably low. A controlled reaction behavior can therefore be concluded. For high comonomer concentrations, the $\oslash$-values increase above values which are typical for RAFT star polymerizations (see below). As with BA, a rate reduction by the addition of $\mathbf{1 . 1}$ takes place (see Figure 8), which is 
even more pronounced in the case of NIPAm than for BA. This may be due to the differences in the parent structure of the monomers 1.1 and NIPAm, i.e., acrylate $v$ s. acrylamide.

Figure 7. Evolution of the number average molecular weight, $M_{\mathrm{n}}$, and dispersity, $\oslash$, with the monomer conversion for a homopolymerization of NIPAm (full symbols) and various copolymerizations of NIPAm and $\mathbf{1 . 1}$ using $0.6 \mathrm{mmol} \cdot \mathrm{L}^{-1} \mathbf{R}_{\mathbf{6}} \mathbf{3}$ and $1.1 \mathrm{mmol} \cdot \mathrm{L}^{-1} \mathrm{AIBN}$ in DMF at $60{ }^{\circ} \mathrm{C}$.

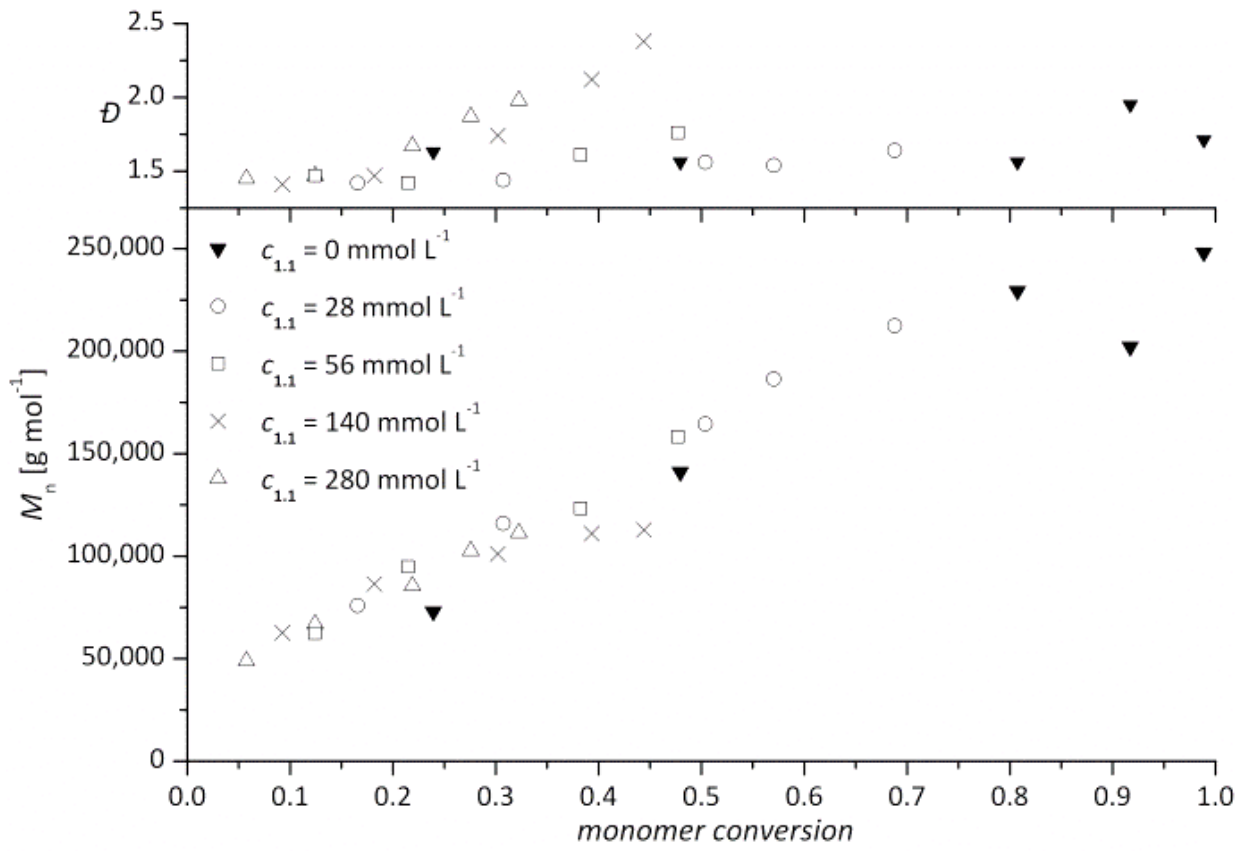

Figure 8. Development of the number average molecular weight, $M_{\mathrm{n}}$, and the monomer conversion with the reaction time for a homopolymerization of NIPAm (filled symbols) and various copolymerizations of NIPAm and $\mathbf{1 . 1}$ using $0.6 \mathrm{mmol} \cdot \mathrm{L}^{-1} \mathbf{R}_{\mathbf{6}} \mathbf{3}$ and $1.1 \mathrm{mmol} \cdot \mathrm{L}^{-1}$ AIBN in DMF at $60{ }^{\circ} \mathrm{C}$.

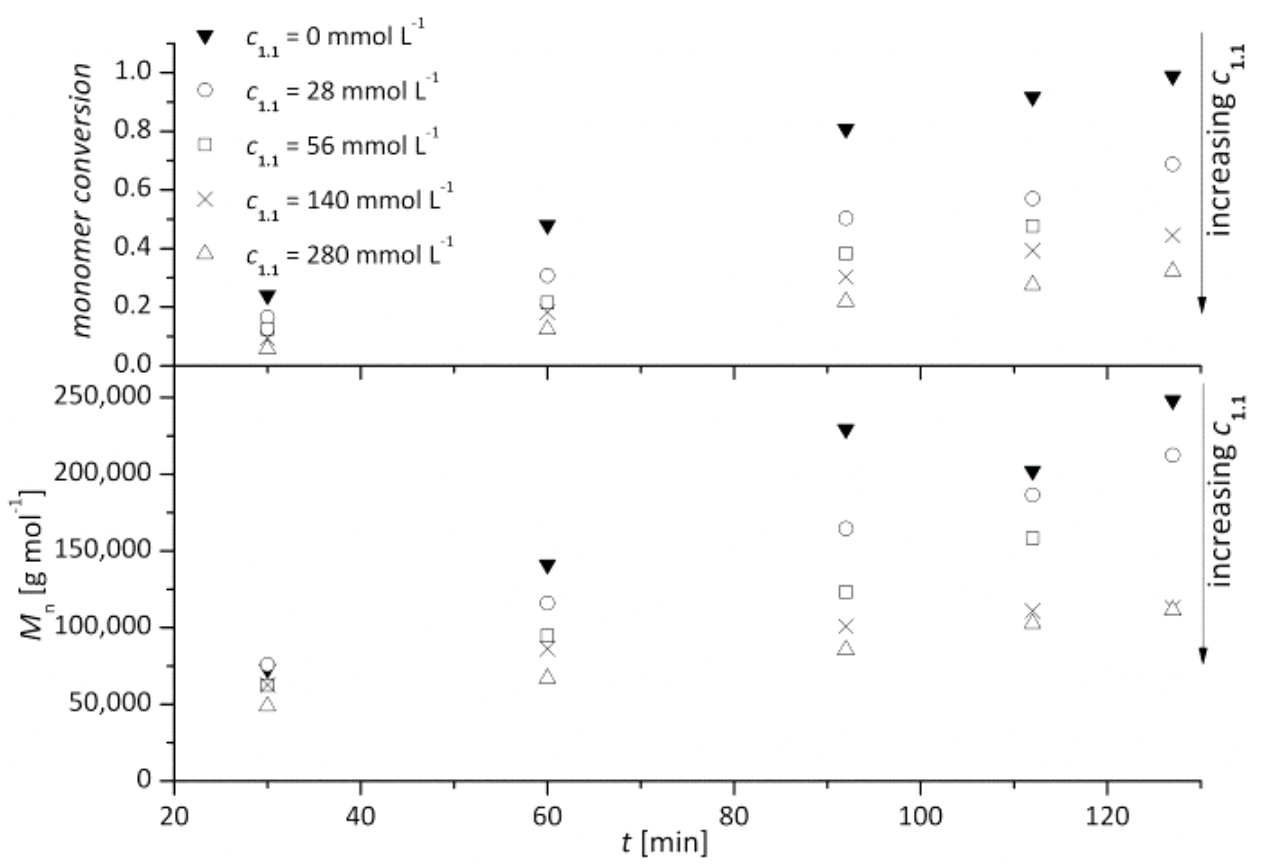


Similar to the BA-polymerization, it can be observed that the effect of rate reduction is more pronounced for systems with higher comonomer loads. Comparing the chromatograms of aliquots taken after the same period of time (Figure 9) consequently shows a decrease in the molecular weight with increasing comonomer concentration.

Figure 9. Molar mass distributions (SEC, RI) from different copolymerizations of NIPAm with 1.1 using $0.6 \mathrm{mmol} \cdot \mathrm{L}^{-1} \mathbf{R}_{\mathbf{6}} \mathbf{3}$ and $1.1 \mathrm{mmol} \cdot \mathrm{L}^{-1} \mathrm{AIBN}$ in $\mathrm{DMF}$ at $60{ }^{\circ} \mathrm{C}$ after $30 \mathrm{~min}(\mathbf{a})$ and $127 \mathrm{~min}(\mathbf{b})$.

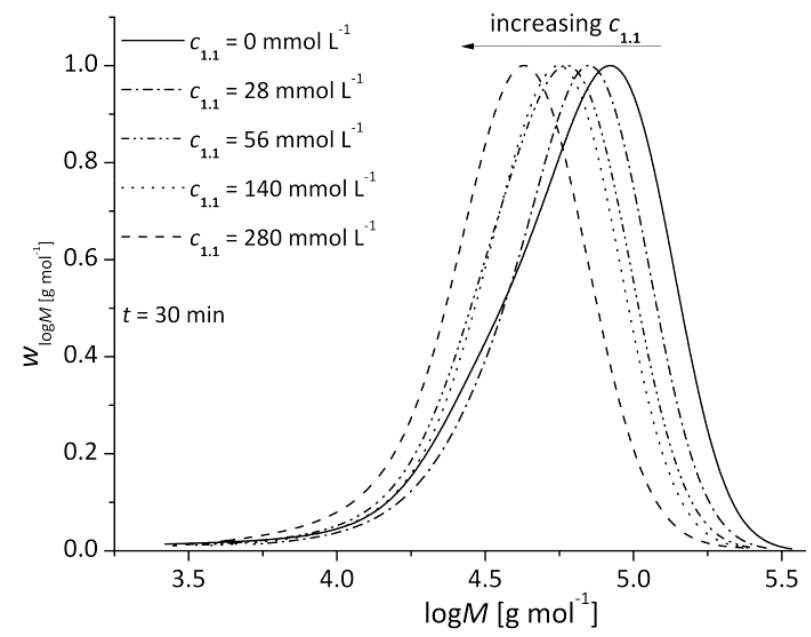

(a)

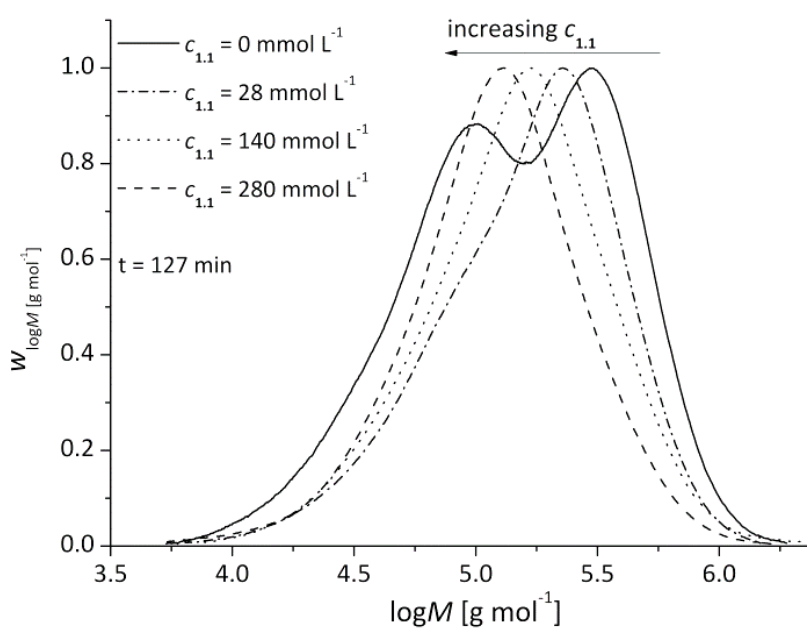

(b)

In contrast to the corresponding chain length distributions from copolymerizations with BA, no clear bimodalities with shoulders in the region of high molecular weights were observed. This indicates that hardly any star-star coupling occurs in Z-RAFT star polymerizations of NIPAm. The poly-NIPAm macroradical is apparently not active enough to attack the dimethylmaleimide-moiety.

The chromatograms obtained by RI-detection (Figure 10), however, are broad and partially bimodal with peaks occurring at the low molar mass side, due to dead polymer chains generated in the polymerization process. The effect is most prominent in systems without any $\mathbf{1 . 1}$ (see Figure 10). Information can again be drawn from UV detection after SEC: Whereas the UV-data, which is only sensitive to polymer carrying RAFT groups (stars in this case), indicates a unimodal distribution, it can be seen that a large amount of UV-inactive material, i.e., dead polymer without RAFT-moieties, is present at low molar masses resulting in high overall $\emptyset$-values from RI-detection (see Figure 10). The formation of dead polymer material increases with reaction time. It thus appears that termination is rather high in this system, which, however, is in conflict with findings by Gilbert et al., who qualitatively found that termination in aqueous NIPAm polymerization is low [35]. As propagation in acrylate and NIPAm polymerization is similar [36,37] — note that a slow propagation may also lead to high amounts of terminated chains after the same extent of monomer conversion-lower amounts of terminated RAFT polymer would thus be anticipated according to Gilbert's findings, which clearly is not the case. It is therefore more likely that some other mechanism than biradical termination leads to deactivation of chains under loss of the RAFT-units. The acrylamide-type monomer NIPAm may form small amounts of amines via hydrolysis, which in turn is known to cleave RAFT-polymer [38]. The hydrolysis of amides in neutral water is known to occur slowly with a rate coefficient of $3 \cdot \times 10^{9} \mathrm{~s}^{-1}$ [39], 
which is, however, sufficient to produce free amines in the order of tenths of millimoles in these highly concentrated NIPAm solutions after the respective reaction times. The same level of concentration was chosen for the star RAFT agents, which may thus be decomposed significantly. The result of this process between amines and thiocarbonylthio-moieties is also a continuous formation of RAFT-group-lacking arm polymer, equal to the material that may form via termination. High amounts of dead polymer, which constitute arm polymer chains that have been separated from the star polymer, imply that significant amounts of the star polymer have lost at least one of their arms. The assumption for the UV-trace correction that all macromolecules carry the same amounts of absorbing groups is thus not fully valid any longer, which may affect a potential quantitative analysis using both RI- and corrected UV-traces. The qualitative conclusions drawn in the present work are, however, not affected by this small uncertainty.

Figure 10. Chromatograms of aliquots of the NIPAm homopolymerization using $0.6 \mathrm{mmol} \cdot \mathrm{L}^{-1} \mathbf{R}_{\mathbf{6}} \mathbf{3}$ and $1.1 \mathrm{mmol} \cdot \mathrm{L}^{-1} \mathrm{AIBN}$ in DMF at $60{ }^{\circ} \mathrm{C}$ after different periods of time (full $=$ RI-data, dashed $=$ corrected UV-data).

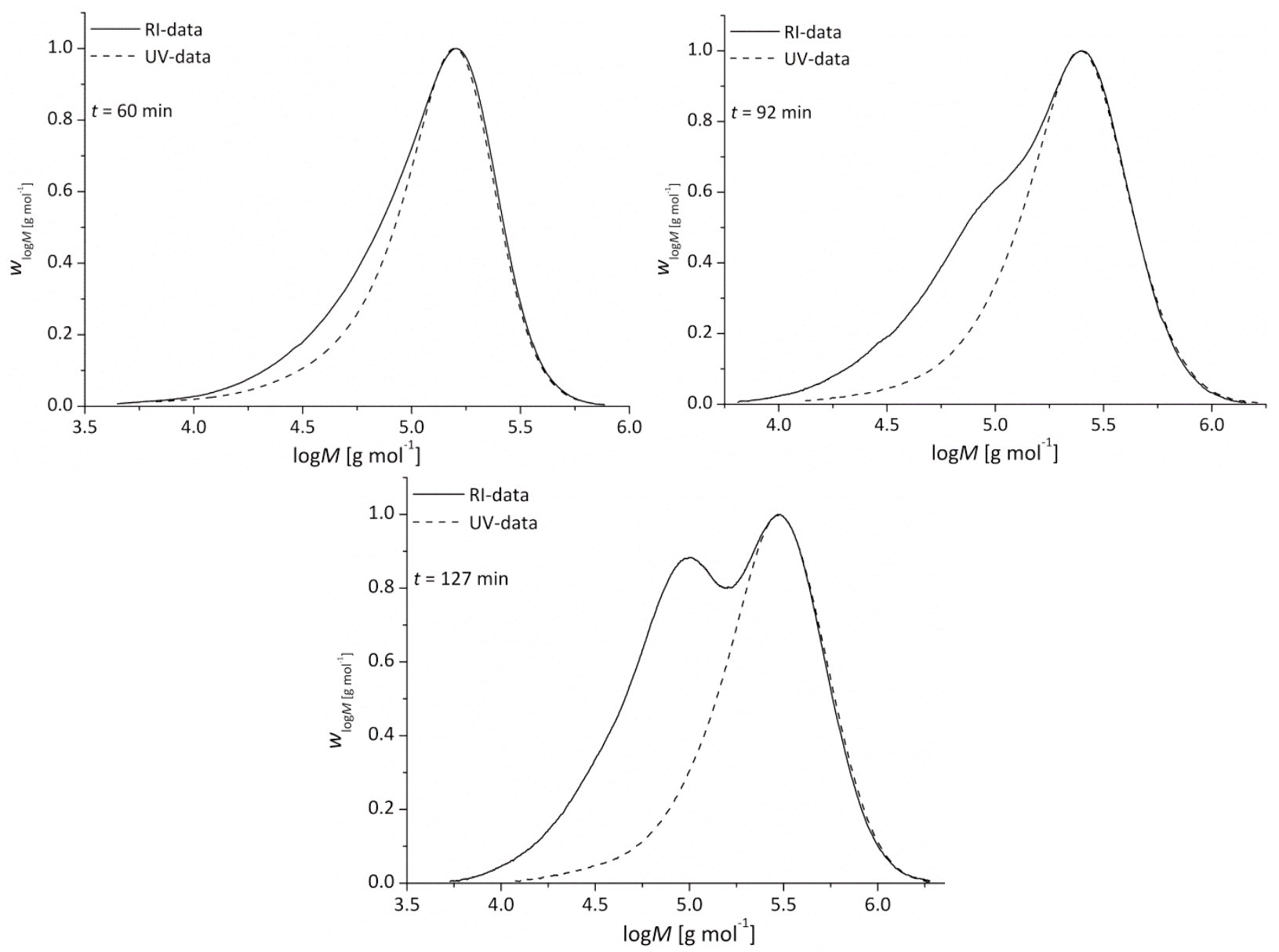

In Figure 11, the chromatograms determined by RI-detection of copolymerizations with various amounts of $\mathbf{1 . 1}$ are shown. It is interesting to note that the fraction of dead arm polymer at the low molar mass side of the star polymer is highest in the homopolymerization of NIPAm and is getting less prominent when increasing the concentration of $\mathbf{1 . 1}$ as comonomer. This finding is in line with the above interpretation about RAFT degradation via monomer-derived amines: when using lower 
concentrations of NIPAm, as is the case in copolymerization, less amounts of amines are expected, i.e., star degradation may proceed with lower rates. In any case, very homogenous copolymers carrying photocrosslinkable croups are obtained via the chosen strategy.

Figure 11. Chain length distributions (SEC, RI-detection) of copolymerizations of NIPAm with 1.1 using $0.6 \mathrm{mmol} \cdot \mathrm{L}^{-1} \mathbf{R}_{\mathbf{6}} 3$ and $1.1 \mathrm{mmol} \cdot \mathrm{L}^{-1} \mathrm{AIBN}$ in DMF at $60{ }^{\circ} \mathrm{C}$ up to a reaction time of $127 \mathrm{~min}$.

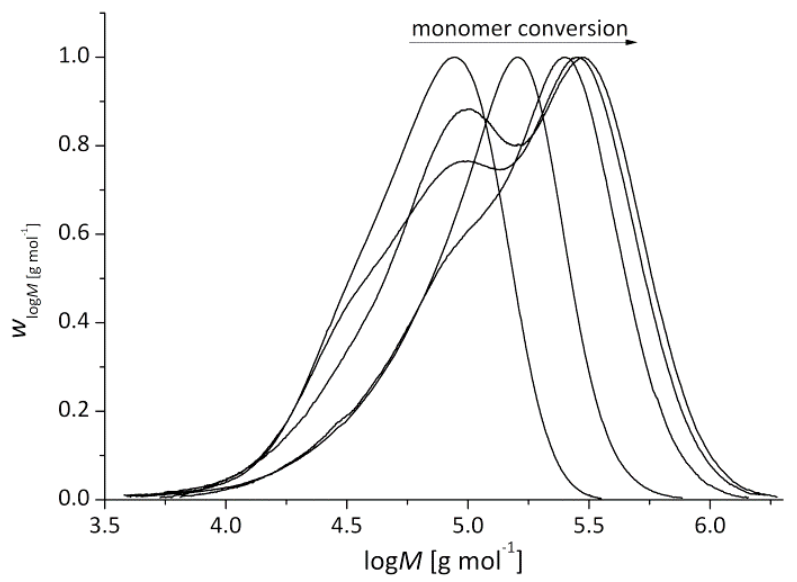

Homopolymerization of NIPAm

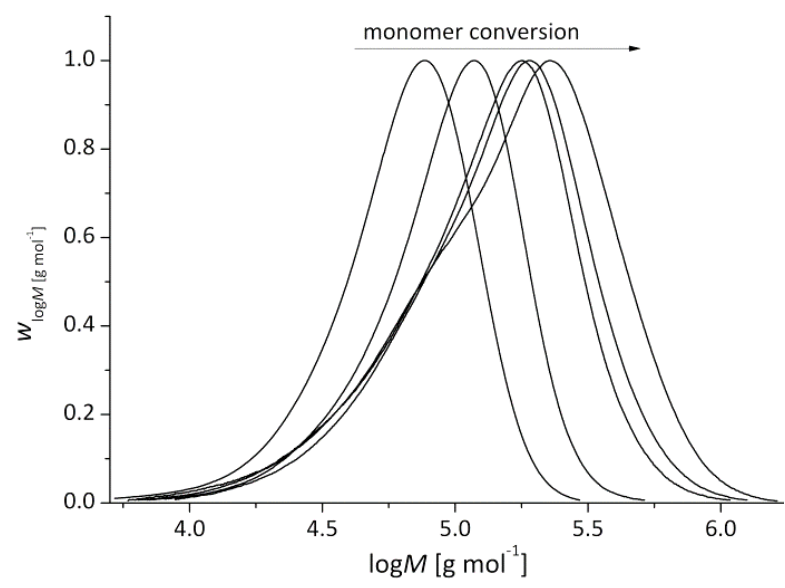

Copolymerization with $28 \mathrm{mmol} \cdot \mathrm{L}^{-1} \mathbf{1 . 1}$

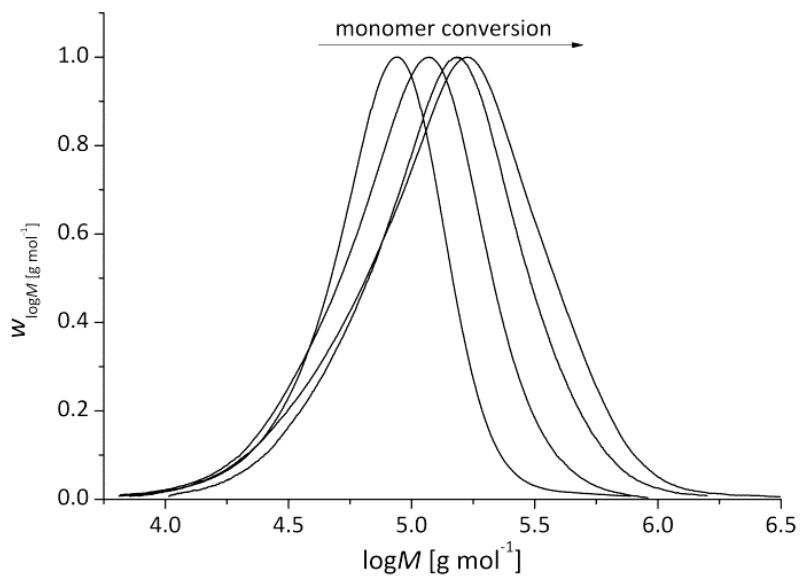

Copolymerization with $140 \mathrm{mmol} \cdot \mathrm{L}^{-1} \mathbf{1 . 1}$

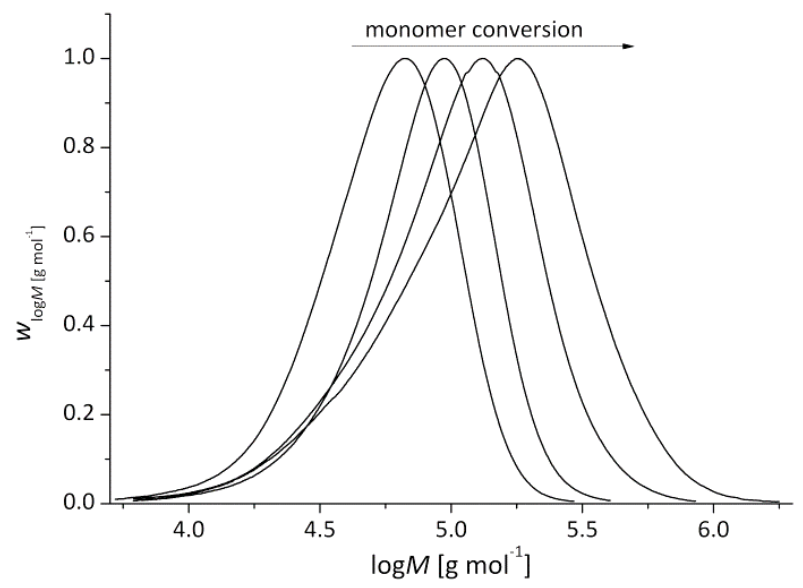

Copolymerization with $56 \mathrm{mmol} \cdot \mathrm{L}^{-1} \mathbf{1 . 1}$

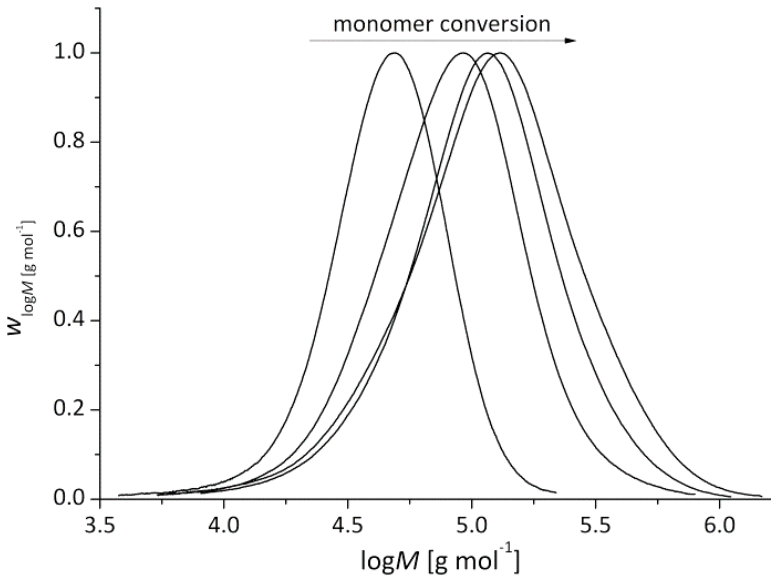

Copolymerization with $280 \mathrm{mmol} \cdot \mathrm{L}^{-1} \mathbf{1 . 1}$ 
Next to the formation of dead polymer at the low molar mass side, a small broadening effect originating from star-star coupling was observed at high molar masses. When inspecting the living material via UV-detection (see Figure 12), it can be seen that at prolonged reaction times and at high concentrations of 1.1, small amounts of star couples occur. In the last set of chromatograms of the copolymerization with the highest comonomer concentration the peak maxima are marked. They differ roughly by a factor of 2 , i.e., indicating that a very small amount of double stars are formed. However, the formation of high molecular weight material is clearly not as pronounced as in the copolymerizations with BA.

Figure 12. Corrected UV-SEC-curves of the copolymerizations of NIPAm with $\mathbf{1 . 1}$ up to a reaction time of $127 \mathrm{~min}$. (a) $140 \mathrm{mmol} \cdot \mathrm{L}^{-1}$; (b) $280 \mathrm{mmol} \cdot \mathrm{L}^{-1}$.

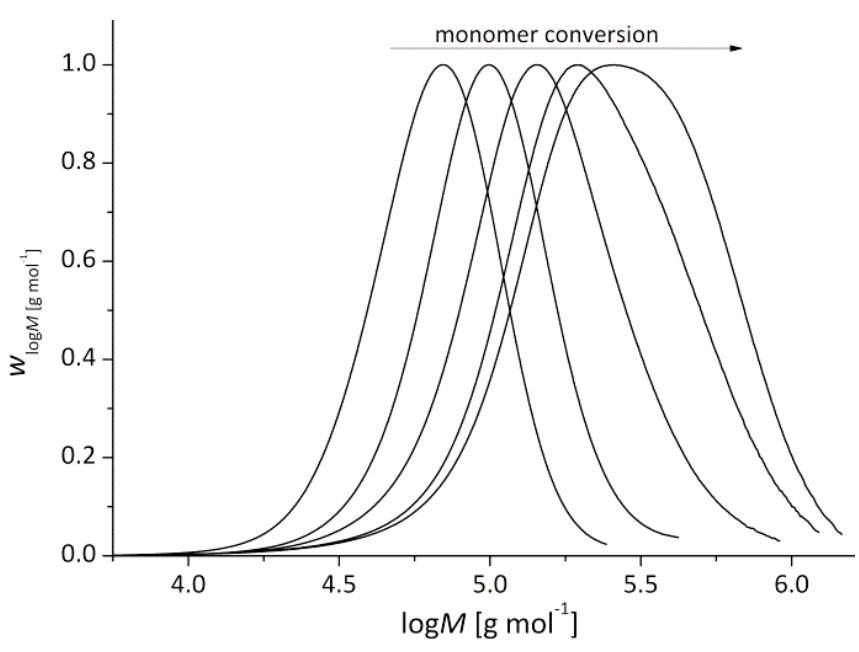

(a)

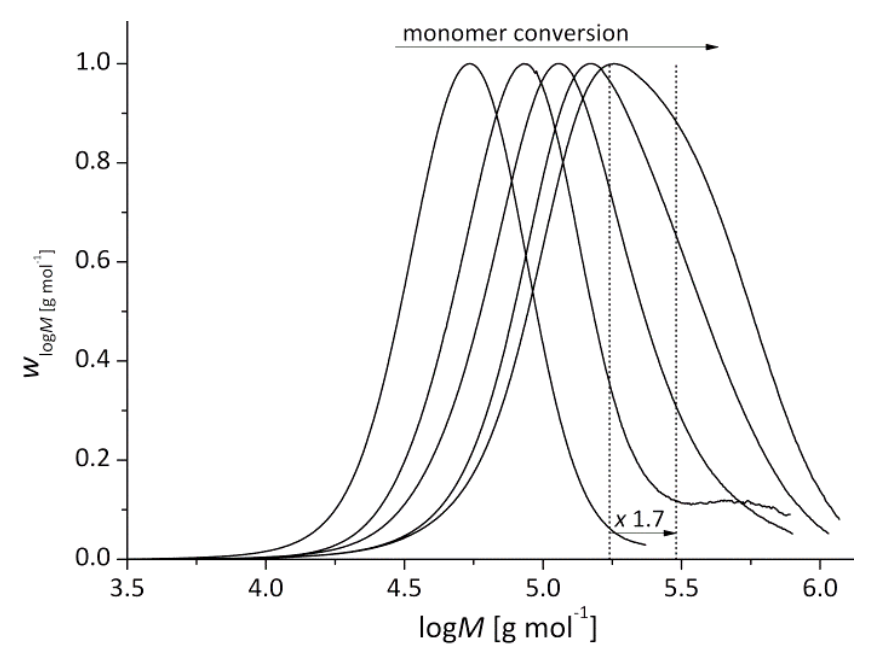

(b)

\subsection{Determination of the Copolymer Composition}

A special interest of this work was determining the copolymer composition of the product. The here studied star polymers that serve as template for nano-carriers are containing polymer blocks consisting of two statistically distributed monomers, one of which carries the photocrosslinkable group. NMR spectroscopy was used to determine the composition of the copolymer. A standard method for this is the comparison of the integrals of specific signals belonging to the various monomer-moieties which build up the polymer [40]. A requirement to apply this method is the full characterization of the comonomer 1.1.

In order to gather connectivity information between $\mathrm{N}$ and $\mathrm{H}$ and to check whether the concentrations of the different compounds in the system are sufficient, ${ }^{15} \mathrm{~N}-\mathrm{HMBC}$ experiments were carried out with the reaction mixture $\left(c_{1.1}=105 \mathrm{mmol} \cdot \mathrm{L}^{-1}, c_{\mathrm{AIBN}}=0.85 \mathrm{mmol} \cdot \mathrm{L}^{-1}, c_{\mathbf{R 6 2}}=0.55 \mathrm{mmol} \cdot \mathrm{L}^{-1}\right.$ in a toluene: BA mixture $1: 1$, equals $c_{\mathrm{BA}}=351 \mathrm{mmol} \cdot \mathrm{L}^{-1}$ ). The ${ }^{1} \mathrm{H}$ NMR spectrum of this mixture is shown in Figure S3 of the ESI. Figure 13 shows the ${ }^{15} \mathrm{~N}-\mathrm{HMBC}$ of this polymerization mixture. On the $y$-scale, the ${ }^{15} \mathrm{~N}$ NMR spectrum, and on the $\mathrm{x}$-scale, the ${ }^{1} \mathrm{H}$ NMR spectrum (Figure S3 in ESI) are plotted. The observed cross-peaks are the result of a transfer of magnetization between the ${ }^{1} \mathrm{H}$ and ${ }^{15} \mathrm{~N}$ nuclei in proximity to each other. Interactions between two cores generally occur via one to three bonds so that structural conclusions could be drawn by these cross-peaks. 
Figure 13. ${ }^{15} \mathrm{~N}-\mathrm{HMBC}$ spectrum of the reaction mixture recorded in $\mathrm{CDCl}_{3}\left({ }^{15} \mathrm{~N}\right.$ versus $\left.{ }^{1} \mathrm{H}\right)$.

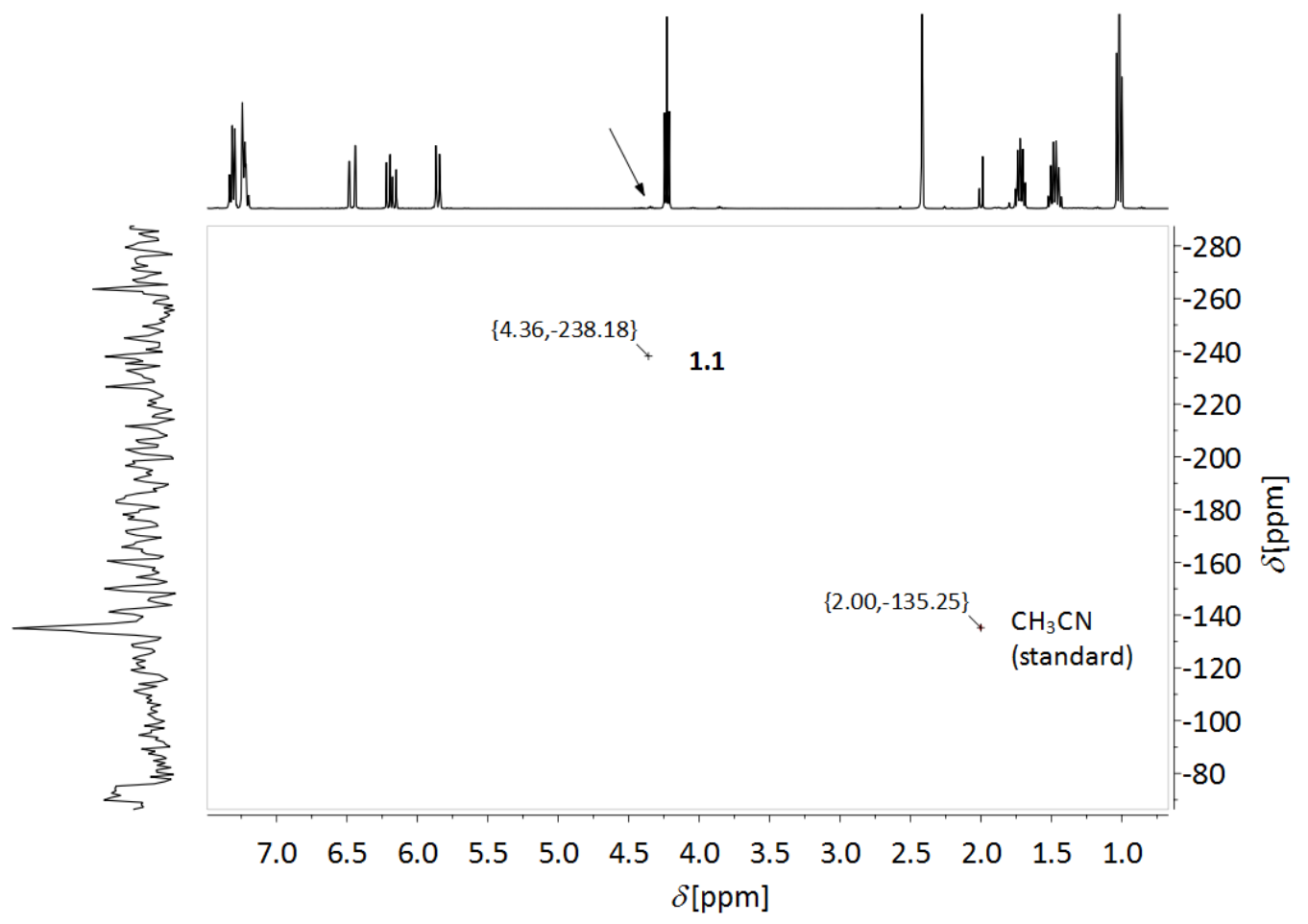

The chemical shift of $\mathbf{1 . 1}$ in the ${ }^{15} \mathrm{~N}-\mathrm{HMBC}$ was determined to be $4.36 \mathrm{ppm}$ in the ${ }^{1} \mathrm{H}$ dimension and $-238.18 \mathrm{ppm}$ in the ${ }^{15} \mathrm{~N}$ dimension. For acetonitrile values of $2.00 \mathrm{ppm}$ and $-135.25 \mathrm{ppm}$ were found which are confirmed by the literature [41]. Accordingly, the triplet in the upfield region at approximately $3.8 \mathrm{ppm}$ should result from the protons of the $\mathrm{CH}_{2}$-group directly adjacent to the oxygen atom. H,H-COSY measurements confirm this statement. The corresponding H,H-COSY plot is shown in Figure S4 of the ESI. Based on the cross-peaks, it could be proven that the protons of the $\mathrm{CH}_{2}$-group adjacent to the nitrogen, which was classified previously in the ${ }^{15} \mathrm{~N}$-HMBC experiment, are interacting with the protons of the $\mathrm{CH}_{2}$-group adjacent to the oxygen atom, i.e., they are directly connected through bonds. With these data in hand, it can be stated that the triplet of the ethyl bridge in 1.1 in the downfield region at $4.36 \mathrm{ppm}$ results from the protons which are directly adjacent to the nitrogen atom of the DMI-moiety. Furthermore the triplet at $3.78 \mathrm{ppm}$ arises from the protons which are directly adjacent to the oxygen atom. This information helps in the determination of the amount of 1.1-units in the copolymer by integration of the peaks. It has become apparent that it is not feasible to use HMBC investigations for the polymer samples as the broadening of the signals caused by the polymer environment often inhibits the detection of the cross-peaks. ${ }^{1} \mathrm{H}$ NMR spectra were recorded and analyzed instead (Figure 14).

Figure 15 shows the superimposed ${ }^{1} \mathrm{H}$ NMR spectra of a copolymerization with $5000 \mathrm{mmol} \cdot \mathrm{L}^{-1} \mathrm{BA}$ and $280 \mathrm{mmol} \cdot \mathrm{L}^{-1} 1.1$ using $0.59 \mathrm{mmol} \cdot \mathrm{L}^{-1} \mathbf{R}_{\mathbf{6}} \mathbf{2}\left(M_{\mathbf{R} 62}=1864.84 \mathrm{~g} \cdot \mathrm{mol}^{-1}\right)$ and $1.09 \mathrm{mmol} \cdot \mathrm{L}^{-1} \mathrm{AIBN}$ in toluene at $60^{\circ} \mathrm{C}$. 
Figure 14. Extract from the ${ }^{1} \mathrm{H}$ NMR spectrum of $p(\mathrm{BA}-c o-1.1)$ in $\mathrm{CDCl}_{3}$.

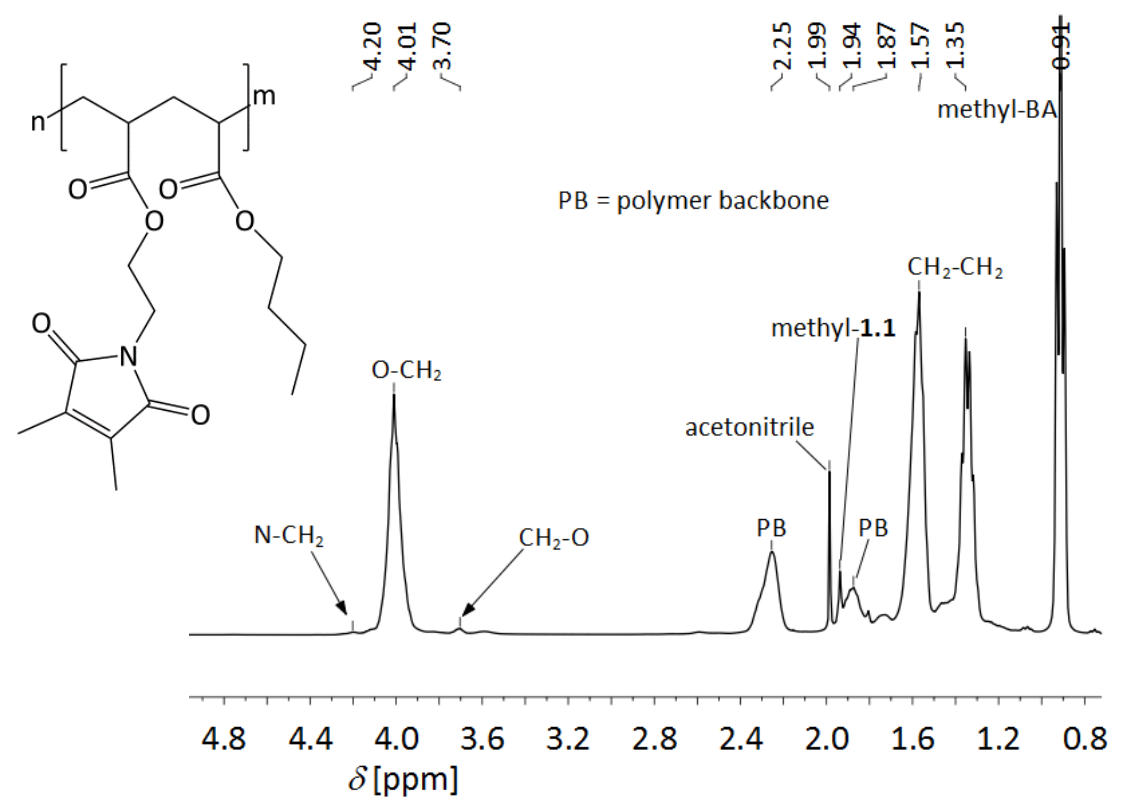

Figure 15. Superimposed, standardized ${ }^{1} \mathrm{H}$ NMR spectra measured in $\mathrm{CDCl}_{3}$ of the first, third and fifth aliquot of a copolymerization of BA and 1.1 using $5000 \mathrm{mmol} \cdot \mathrm{L}^{-1} \mathrm{BA}$, $280 \mathrm{mmol} \cdot \mathrm{L}^{-1} \mathbf{1 . 1}, 0.59 \mathrm{mmol} \cdot \mathrm{L}^{-1} \mathbf{R}_{\mathbf{6}} \mathbf{2}$ and $1.09 \mathrm{mmol} \cdot \mathrm{L}^{-1} \mathrm{AIBN}$ in toluene at $60{ }^{\circ} \mathrm{C}$.

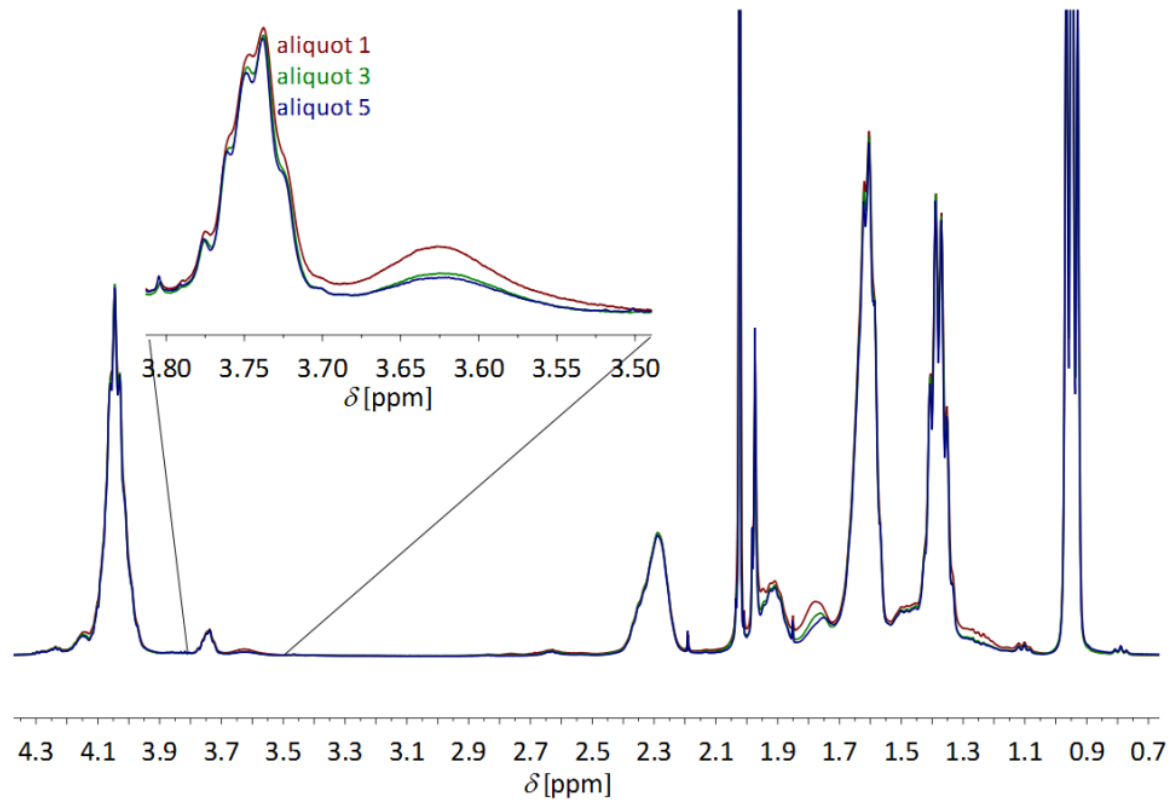

The triplet caused by the methyl group of BA at $0.91 \mathrm{ppm}$ and the signal at $3.70 \mathrm{ppm}$ due to the $\mathrm{CH}_{2}$-group of $\mathbf{1 . 1}$ were integrated. In accordance with the procedure described in literature [40], the integral $\left(I_{\mathrm{BA}}, I_{1.1}\right)$ of the single peaks are referred back to the protons causing the signals. These values are put in relation to each other yielding the molar share $[F(\mathrm{~mol} \%)]$ of each monomer in the polymer. With molecular weights of the monomers $\left(M_{1.1}=223.23 \mathrm{~g} \cdot \mathrm{mol}^{-1}, M_{\mathrm{BA}}=128.17 \mathrm{~g} \cdot \mathrm{mol}^{-1}\right)$, the molecular share is converted to the weight share of each monomer. From the molecular weight of the polymer minus the molecular weight of the RAFT-agent the weight of one star polymer molecule $\left(w_{\text {star }}\right)$ is obtained by applying the Avogadro constant. One star of the first aliquot $\left(M_{\mathrm{n}}=121,000 \mathrm{~g} \cdot \mathrm{mol}^{-1}\right)$ 
without its RAFT-core of the here introduced aliquot has a weight of $1.97 \cdot \times 10^{-19} \mathrm{~g}$. Determining the polymer share of 1.1 with the weight share $(w \% 1.1)$, and divided by the weight of one monomer molecule, the number of monomer units in one star molecule is determined. In the ESI, an example for the calculation of the number of 1.1-units is shown. The calculation for the BA-units was carried out accordingly. In the introduced example 81 1.1-units and 785 BA-units were calculated. Table 2 summarizes the polymer composition in monomer units for the three aliquots of the copolymerization of BA and $\mathbf{1 . 1}$ shown in Figure 15.

Table 2. Determined numbers of BA- and 1.1-units in different polymer samples of a polymerization with $4920 \mathrm{mmol} \cdot \mathrm{L}^{-1} \mathrm{BA}, 280 \mathrm{mmol} \cdot \mathrm{L}^{-1} \mathbf{1 . 1}, 0.59 \mathrm{mmol} \cdot \mathrm{L}^{-1} \mathbf{R}_{\mathbf{6}} \mathbf{2}$ and $1.09 \mathrm{mmol} \cdot \mathrm{L}^{-1} \mathrm{AIBN}$ in toluene at $60{ }^{\circ} \mathrm{C}$.

\begin{tabular}{cccc}
\hline $\boldsymbol{t}(\mathbf{m i n})$ & $\boldsymbol{M}_{\mathbf{n}}\left(\mathbf{g} \cdot \mathbf{m o l}^{\mathbf{- 1}}\right)$ & Number of 1.1-units in the star & Number of BA-units in the star \\
\hline 45 & 121,000 & 81 & 785 \\
115 & 305,000 & 190 & 2,032 \\
155 & 405,000 & 246 & 2,718 \\
\hline
\end{tabular}

In addition to determining the polymer composition by monomer units, the molecular share of a specific monomer in the polymer, $F$, can be used to apply the Lewis-Mayo-equation and obtain the reactivity ratios. To do so, the integral of the BA's methyl group at approximately $0.91 \mathrm{ppm}$ is compared with the integral at $3.70 \mathrm{ppm}$ belonging to the $\mathrm{CH}_{2}$-group of the comonomer, as shown before. Now the molecular composition of the polymer $(F)$ is plotted versus the composition of the monomer feed $(f)$ for all reaction mixtures and fitted via the Lewis-Mayo-equation (Equation (1)) in OriginPro 8.1 giving the reactivity ratios $r_{\mathbf{1 . 1}}$ (a) and $r_{\mathrm{BA}}$ (b) (see Figure 16).

$$
F_{1}=\frac{r_{1} \cdot f_{1}^{2}+f_{1} \cdot f_{2}}{r_{1} \cdot f_{1}^{2}+2 \cdot f_{1} \cdot f_{2}+r_{2} \cdot f_{2}^{2}}
$$

Figure 16. Copolymerization plot for a set of copolymerizations with BA and $\mathbf{1 . 1}$ using $0.59 \mathrm{mmol} \cdot \mathrm{L}^{-1} \mathbf{R}_{\mathbf{6}} \mathbf{2}$ and $0.97 \mathrm{mmol} \cdot \mathrm{L}^{-1} \mathrm{AIBN}$ in toluene at $60{ }^{\circ} \mathrm{C}$ and $90 \mathrm{~min}$ reaction time.

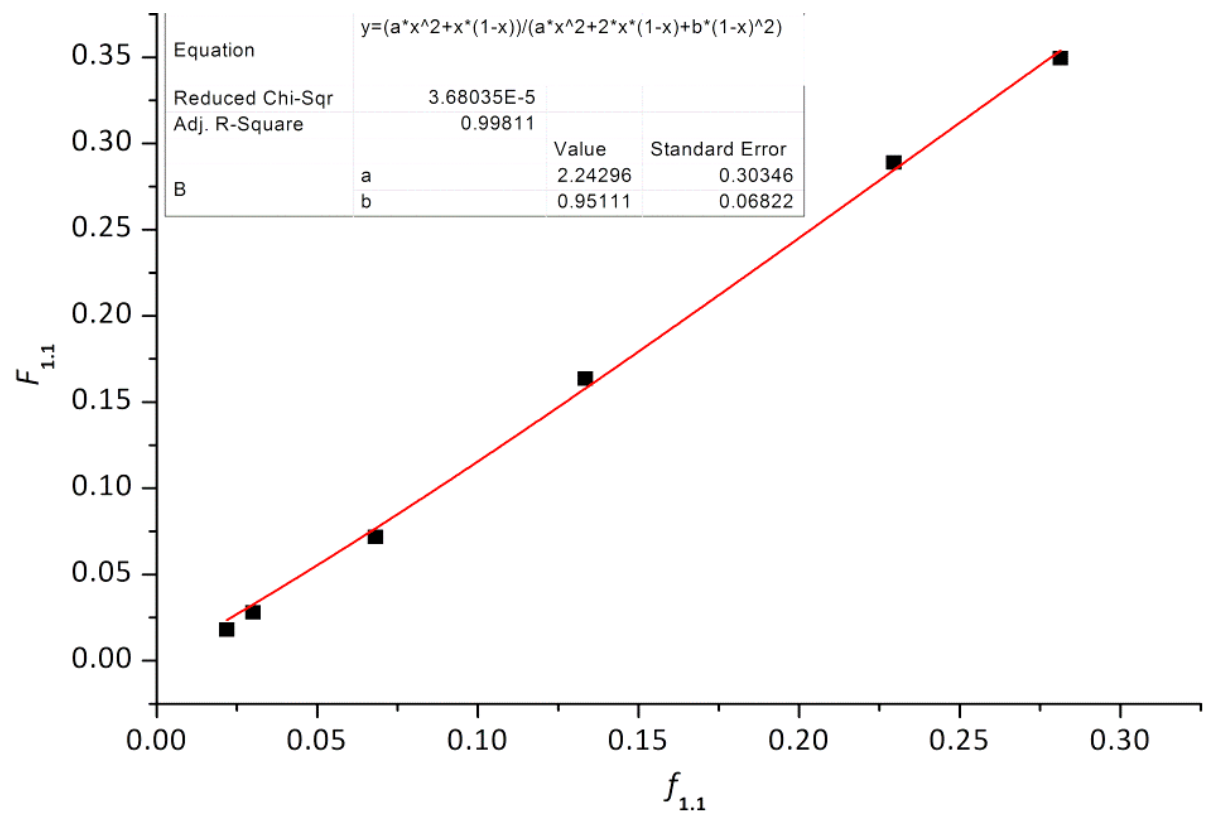


Reactivity ratios of 2.24 for $\mathbf{1 . 1}$ and 0.95 for BA could be determined. Reactivity ratios above 1 are a signal that the growing radicals favor their own monomer, while monomers with reactivity ratios below 1 favor the opposite monomer. Here, the incorporation of the comonomer into the polymer is favored by both types of macroradicals. Thus, $\mathbf{1 . 1}$ is consumed preferably in the beginning. With decreasing concentration of this comonomer in the feed, the probability of consumption eventually also decreases, hence BA is more strongly consumed in later phases of the copolymerization.

By taking a closer look at these reactivity ratios, it can be concluded that a gradient copolymer is formed. This can also be seen from Table 3, in which the copolymer composition is collated for polymer of various chain-lengths. It can be seen that the nominal proportion of $\mathbf{1 . 1}$ is decreasing with increasing chain-length, which is correlated to increasing monomer conversions, due to the controlled nature of the studied system. This drop in $F_{\mathbf{1 . 1}}$ with chain length directly reflects the composition drift due to monomer conversion. As chain length and monomer conversion cannot easily be separated in RAFT polymerization, this composition drift adds to the uncertainty of the absolute values of the reactivity ratios, even when monomer conversions are targeted to be as low as possible.

Table 3. Comparison of the molecular percentage of monomer units in the copolymers.

\begin{tabular}{|c|c|c|c|c|c|}
\hline \multicolumn{3}{|c|}{$\begin{array}{c}\text { Copolymerization with } \\
5000 \mathrm{mmol} \cdot \mathrm{L}^{-1} \text { BA and } 1350 \mathrm{mmol} \cdot \mathrm{L}^{-1} 1.1\end{array}$} & \multicolumn{3}{|c|}{$\begin{array}{c}\text { Copolymerization with } \\
5000 \mathrm{mmol} \cdot \mathrm{L}^{-1} \text { BA and } 280 \mathrm{mmol} \cdot \mathrm{L}^{-1} 1.1\end{array}$} \\
\hline$M_{\mathrm{n}}\left(\mathrm{g} \cdot \mathrm{mol}^{-1}\right)$ & $F_{1.1}(\mathrm{~mol} \%)$ & $F_{\mathrm{BA}}(\mathrm{mol} \%)$ & $M_{\mathrm{n}}\left(\mathrm{g} \cdot \mathrm{mol}^{-1}\right)$ & $F_{1.1}(\mathrm{~mol} \%)$ & $F_{\mathrm{BA}}(\mathrm{mol} \%)$ \\
\hline 88,000 & 37.9 & 62.1 & 120,000 & 9.4 & 90.6 \\
\hline 160,000 & 33.6 & 66.4 & 305,000 & 8.6 & 91.4 \\
\hline 310,000 & 30.1 & 69.9 & 405,000 & 8.3 & 91.7 \\
\hline
\end{tabular}

For NIPAm-based copolymers the procedure was carried out similarly. Figure 17 shows the ${ }^{1} \mathrm{H}$ NMR spectrum of a poly-NIPAm sample recorded in $\mathrm{CDCl}_{3}$. In Figure 18, a ${ }^{1} \mathrm{H}$ NMR spectrum of a copolymer sample is superimposed with a homopolymer sample.

Figure 17. ${ }^{1} \mathrm{H}$ NMR spectrum of poly-NIPAm recorded in $\mathrm{CDCl}_{3}$.

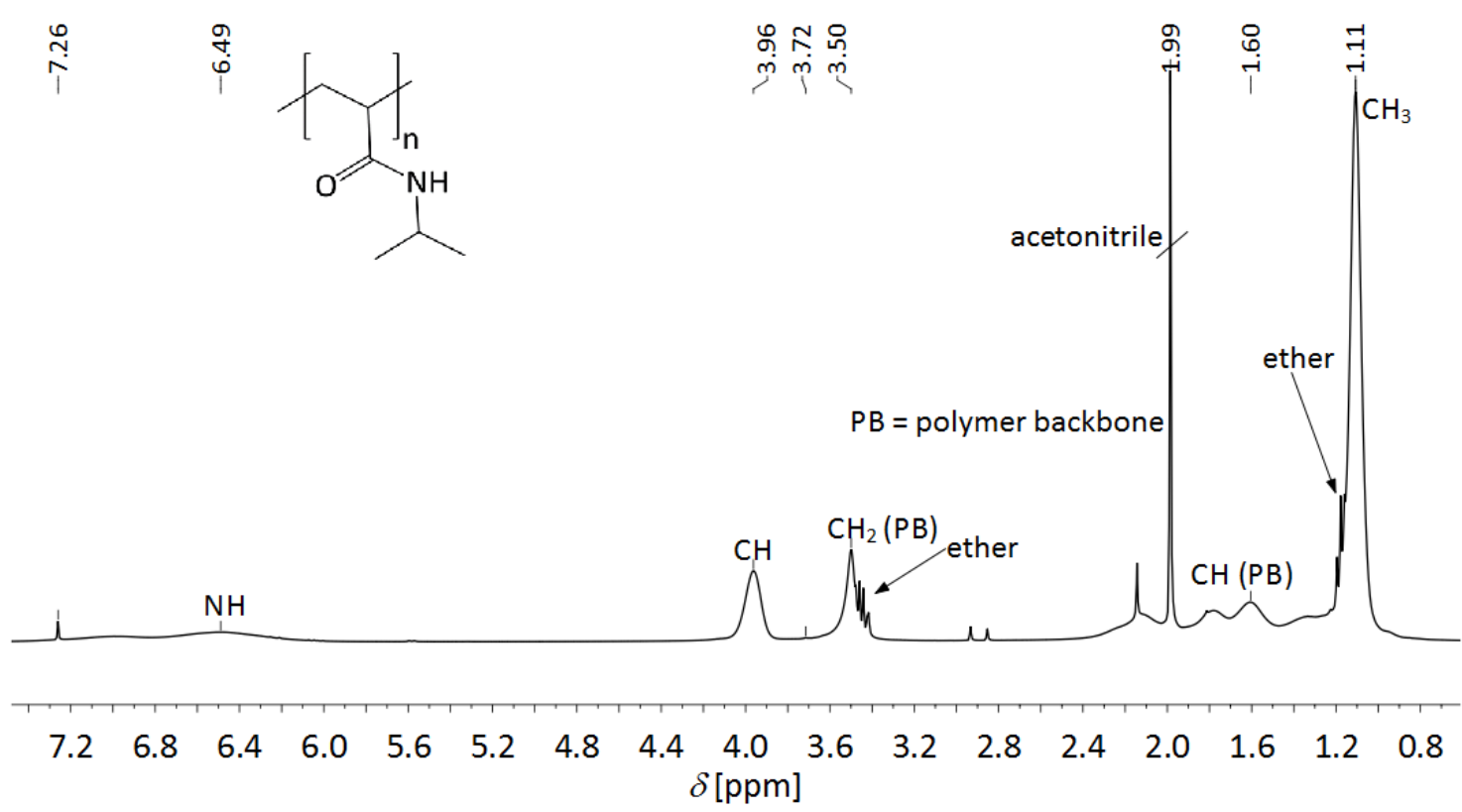


Figure 18. Superimposed, standardized ${ }^{1} \mathrm{H}$ NMR spectra of poly-NIPAm (blue) and poly-(NIPAm-co-1.1) (black) recorded in $\mathrm{CDCl}_{3}$.

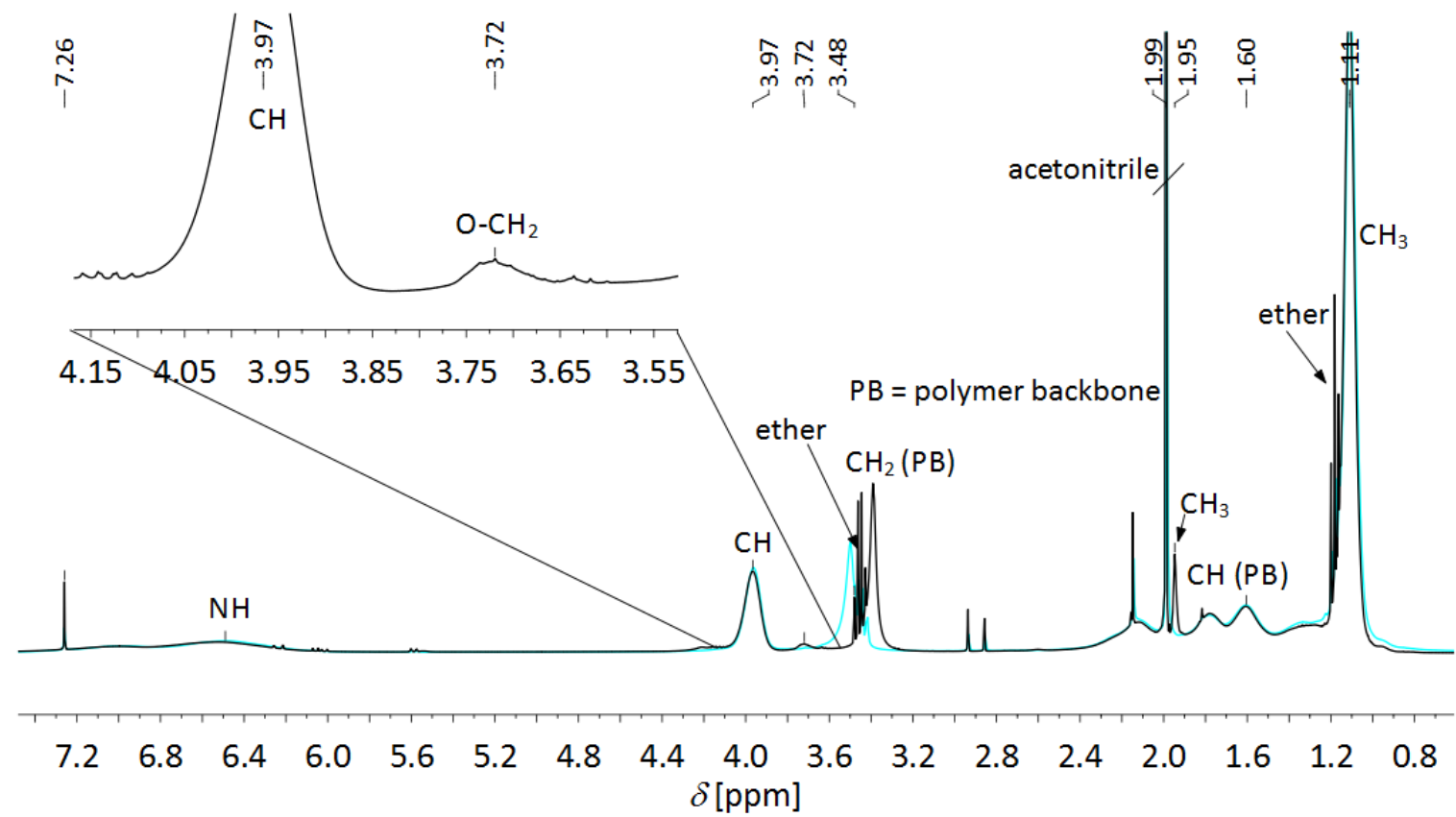

Figure 19. Superimposed, standardized ${ }^{1} \mathrm{H}$ NMR spectra recorded in $\mathrm{CDCl}_{3}$ of the copolymerization with $5,000 \mathrm{mmol} \cdot \mathrm{L}^{-1} \mathrm{NIPAm}$ and $280 \mathrm{mmol} \cdot \mathrm{L}^{-1} \mathbf{1 . 1} \mathrm{using} 0.6 \mathrm{mmol} \cdot \mathrm{L}^{-1}$ $\mathbf{R}_{\mathbf{6}} 3$ and $1.1 \mathrm{mmol} \cdot \mathrm{L}^{-1}$ AIBN in DMF at $60^{\circ} \mathrm{C}$.
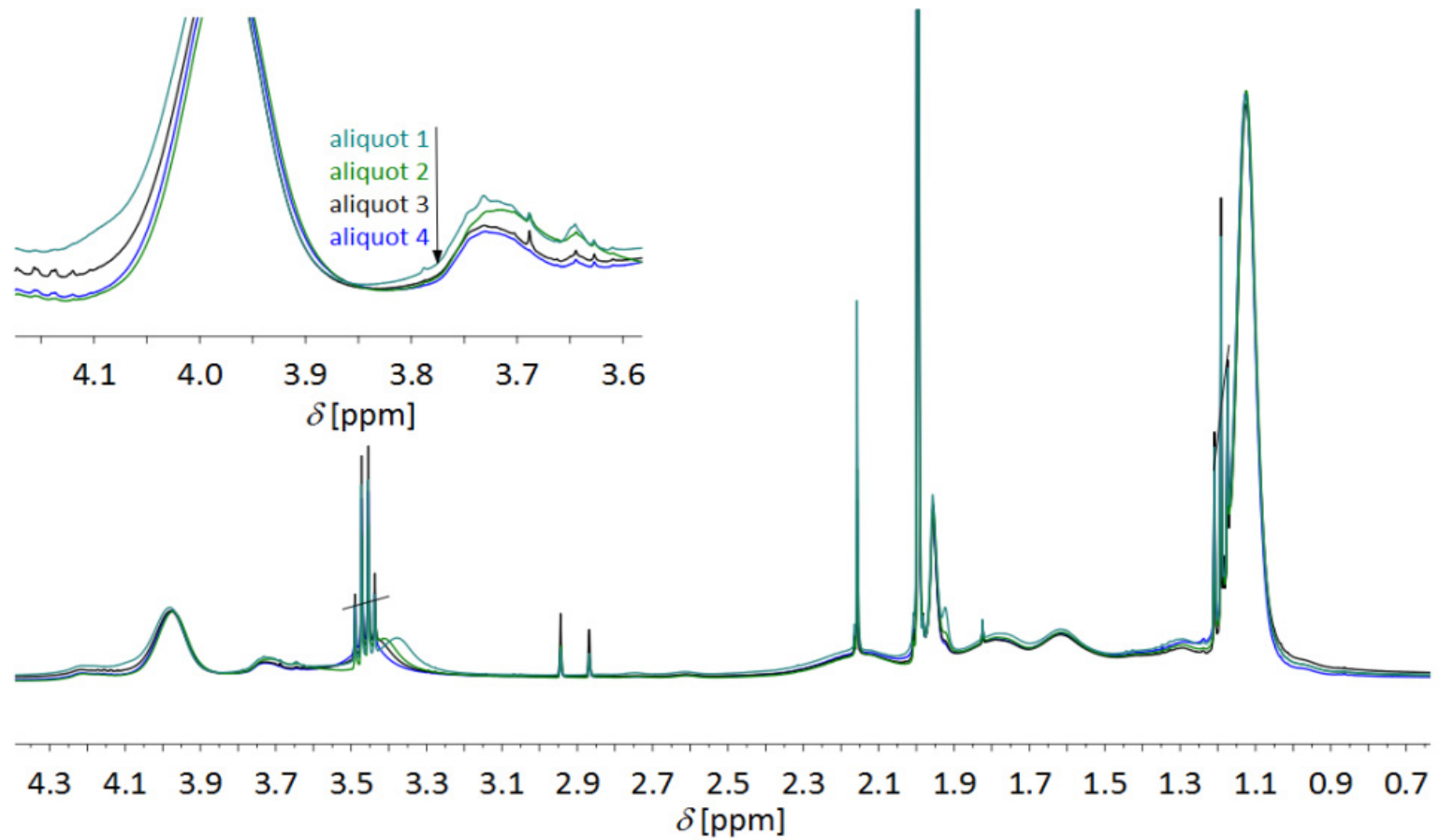

The addition of the comonomer 1.1 causes a singlet at $1.95 \mathrm{ppm}$ resulting from the methyl groups of the dimethylmaleimide-unit and a signal at $3.72 \mathrm{ppm}$, which stems from the $\mathrm{CH}_{2}$-group adjacent to the oxygen atom of the ethyl bridge in the comonomer which was classified by ${ }^{15} \mathrm{~N}-\mathrm{HMBC}$ before. The second signal of the ethyl bridge is overlapped by the large signal of NIPAm's CH-group. Signals of 
diethyl ether, the precipitant for poly-NIPAm, were found in all spectra despite drying the samples for five days under vacuum at $50{ }^{\circ} \mathrm{C}$. For the determination of the molecular composition of these polymers, the integrals of the half-peak widths of the singlet at $1.11 \mathrm{ppm}$ caused by NIPAm's terminal methyl groups and of the comonomer signal at $3.72 \mathrm{ppm}$ were used as described earlier. In an example of a copolymerization between NIPAm and $1.1\left(3600 \mathrm{mmol} \cdot \mathrm{L}^{-1}\right.$ of NIPAm with $107.2 \mathrm{mmol} \cdot \mathrm{L}^{-1}$ of 1.1 using $1.3 \mathrm{mmol} \cdot \mathrm{L}^{-1}$ of AIBN and $0.5 \mathrm{mmol} \cdot \mathrm{L}^{-1}$ of $\mathbf{R}_{6} \mathbf{3}$ in DMF) after $55 \mathrm{~min}$ of reaction time star polymer with $95.2 \mathrm{~mol} \%$ NIPAm and $4.8 \mathrm{~mol} \% \mathbf{1 . 1}$ and after $85 \mathrm{~min}$ with $95.7 \mathrm{~mol} \%$ NIPAm and $4.3 \mathrm{~mol} \% 1.1$ were obtained. From the set of copolymerizations introduced earlier $\left(5000 \mathrm{mmol} \cdot \mathrm{L}^{-1}\right.$ of NIPam and $280 \mathrm{mmol} \cdot \mathrm{L}^{-1}$ of $\mathbf{1 . 1}$ in DMF were polymerized with $0.6 \mathrm{mmol} \cdot \mathrm{L}^{-1}$ of $\mathbf{R}_{6} \mathbf{3}$ and $1.1 \mathrm{mmol} \cdot \mathrm{L}^{-1}$ of $\mathrm{AIBN}$ at $60{ }^{\circ} \mathrm{C}$ ) the molecular composition was determined. Figure 19 shows the superimposed, standardized ${ }^{1} \mathrm{H}$ NMR spectra of this copolymerization.

The area between 3.60 and $4.15 \mathrm{ppm}$ was magnified. From this area it can be observed that the amount of incorporated $\mathbf{1 . 1}$ in the polymer decreases in comparison to the amount of NIPam. This is supported by the molecular share $F$ determined (Table 4).

Table 4. Molecular shares of both monomers and number of monomer units per star molecule in the samples of the copolymerization with $5000 \mathrm{mmol} \cdot \mathrm{L}^{-1} \mathrm{NIPAm}$ and $280 \mathrm{mmol} \cdot \mathrm{L}^{-1} \mathbf{1 . 1} \mathrm{using} 0.6 \mathrm{mmol} \cdot \mathrm{L}^{-1} \mathbf{R}_{\mathbf{6}} 3$ and $1.1 \mathrm{mmol} \cdot \mathrm{L}^{-1} \mathrm{AIBN}$ in DMF at $60{ }^{\circ} \mathrm{C}$.

\begin{tabular}{cccccc}
\hline $\begin{array}{c}\boldsymbol{t} \\
(\mathbf{m i n})\end{array}$ & $\boldsymbol{M}_{\mathbf{n}}\left(\mathbf{g} \cdot \mathbf{m o l}^{-\mathbf{1}}\right)$ & $\boldsymbol{F}_{\text {NIPAm }}$ & $\boldsymbol{F}_{\mathbf{1 . 1}}$ & $\begin{array}{c}\text { Number of NIPAm } \\
\text { units in the star }\end{array}$ & $\begin{array}{c}\text { Number of 1.1 } \\
\text { units in the star }\end{array}$ \\
\hline 30 & 49,000 & 76.0 & 24.0 & 266 & 84 \\
60 & 70,000 & 80.9 & 19.1 & 404 & 95 \\
92 & 86,000 & 83.9 & 16.1 & 548 & 106 \\
112 & 103,000 & 85.1 & 14.9 & 675 & 118 \\
\hline
\end{tabular}

The ratio of 1.1-units to NIPAm-units allows the statement that the incorporation of $\mathbf{1 . 1}$ in the beginning of the polymerization is again favored, similar to the findings in the BA-polymerizations. Reactivity ratios were obtained in accordance to the procedure shown before. Values of $r_{1.1}=0.96$ and $r_{\text {NIPAm }}=0.05$ were determined, indicating that the resulting polymer is close to an alternating copolymer. As $\mathbf{1 . 1}$ has a reactivity ratio of almost 1 and NIPAm strongly favors the comonomer, it can be concluded that $\mathbf{1 . 1}$ is preferably integrated as long as the local concentration is high enough.

\section{Conclusions}

This paper describes the Z-RAFT-star copolymerization of BA and NIPAm with $N$-ethylacrylate3,4-dimethylmaleimide (1.1), a monomer carrying a UV-reactive DMI-unit. Experiments with BA and NIPAm showed that the addition of $\mathbf{1 . 1}$ slows down the polymerization rate. The effect increases with increasing comonomer concentration. In the case of copolymerizations with BA, it was found that the comonomer reinforces double star formation. It was proposed that the coupling of stars proceeds via a singular propagation step between a DMI-unit and a growing macroradical. Such coupling reactions were significantly less pronounced in copolymerizations with NIPAm. In the NIPAm systems, however, a strong formation of dead material during the polymerization process leads to smaller molecular weights than expected and to broadening of the molar mass distributions. 
Furthermore, the composition of the copolymers as well as the reactivity ratios for the applied comonomers were determined via NMR spectroscopy $\left(\mathrm{BA}-c o-\mathbf{1 . 1} r_{\mathbf{1 . 1}}=2.24 r_{\mathrm{BA}}=0.95\right.$; NIPAm-co-1.1 $\left.r_{1.1}=0.96 r_{\text {NIPAm }}=0.05\right)$. In both cases the comonomer is consumed preferably in the beginning of the polymerization. It can be concluded that gradient copolymer stars are formed carrying the UV-reactive units in the outer sphere; a fact that can be considered as beneficial for the formation of nano-carriers. The present system is thus excellently suited for the generation of peripherally crosslinked star polymers.

The functional copolymer stars presented here may be used in a subsequent polymerization forming amphiphilic star block copolymers that carry photocrosslinkble groups in the outer sphere. These may form nano-carriers after intramolecular crosslinking by UV-light, which closes the carrier's shell [6]. The functional stars may also form model networks when crosslinked under high concentration conditions. These and other applications are currently explored.

\section{Acknowledgments}

The authors thank the German Research Council (DFG; VA226/3-2) for the financial support. Support from the State of Lower Saxony ( $\mathrm{PhD}$ program Catalysis for Sustainable Synthesis (CaSuS)) is gratefully acknowledged. P.V. acknowledges receipt of a Heisenberg-Professorship (DFG).

\section{References and Notes}

1. Moad, G.; Rizzardo, E.; Thang, S.H. Living radical polymerization by the RAFT process. Aust. J. Chem. 2005, 56, 379-410.

2. Liu, J.; Duong, H.; Whittaker, M.R.; Davis, T.P.; Boyer, C. Synthesis of Functional Core, Star Polymers via RAFT Polymerization for Drug Delivery Applications. Macromol. Rapid Commun. 2012, 33, 760-766.

3. Boyer, C.; Teo, T.; Phillips, P.; Erlich, R.B.; Sagnella, S.; Sharbeen, G.; Dwarte, T.; Duong, H.T.T.; Goldstein, D.; Davis, T.P.; Kavallaris, M.; McCarroll, J. Effective Delivery of siRNA into Cancer Cells and Tumors Using Well-Defined Biodegradable Cationic Star Polymers. Mol. Pharm. 2013, 10, 2435-2444.

4. Moad, G.; Rizzardo, E.; Thang, S.H. Living radical polymerization by the RAFT process -A first update. Aust. J. Chem. 2006, 58, 669-692.

5. Moad, G.; Rizzardo, E.; Thang, S.H. Living radical polymerization by the RAFT process-A second update. Aust. J. Chem. 2009, 62, 1402-1472.

6. Förster, N.; Vana, P. Nano-Carrier Synthesis via Z-RAFT Star Polymerisation. 2013, to be submitted for publication.

7. Parrott, M.C.; Luft, J.C.; Byrne, J.D.; Fain, J.H.; Napier, M.E.; DeSimone, J.M. Tunable Bifunctional Silyl Ether Cross-Linkers for the Design of Acid-Sensitive Biomaterials. J. Am. Chem. Soc. 2010, 132, 17928-17932.

8. Lv, L.-P.; Xu, J.-P.; Liu, X.-S.; Liu, G.-Y.; Yang, X.; Ji, J. Disulfide-Crosslinked Biomimetic Micelles: Formation, Thiol Reactivity and Cytotoxicity Behavior. Macromol. Chem. Phys. 2010, $211,2292-2300$. 
9. Kolb, H.C.; Finn, M.G.; Sharpless, K.B. Click chemistry: Diverse chemical function from a few good reactions. Angew. Chem. Int. Ed. 2001, 40, 2004-2021.

10. Lv, W.; Liu, L.; Luo, Y.; Wang, X.; Liu, Y. Biotinylated thermoresponsive core cross-linked nanoparticles via RAFT polymerization and "click" chemistry. J. Colloid Interface Sci. 2011, 356, 16-23.

11. Kafouris, D.; Patrickios, C.S. Synthesis and characterization of shell-cross-linked polymer networks and large-core star polymers: Effect of the volume of the cross-linking mixture. Eur. Polym. J. 2009, 45, 10-18.

12. Zheng, G.; Pan, C. Preparation of star polymers based on polystyrene or polystyrene-b- $N$-isopropyl acrylamide) and divinylbenzene via reversible addition-fragmentation chain transfer polymerization. Polymer 2005, 46, 2802-2810.

13. Drockenmuller, E.; Li, L.Y.T.; Rye, D.Y.; Harth, E.; Russell, T.P.; Kim, H.-C.; Hawker, C.J. Covalent stabilization of nanostructures: Robust block copolymer templates from novel thermoreactive systems. J. Polym. Sci. A Polym. Chem. 2005, 43, 1028-1037.

14. Yu, X.; Corten, C.; Görner, H.; Wolff, T.; Kuckling, D. Photodimers of $\mathrm{N}$-alkyl-3,4-dimethylmaleimides_-Product ratios and reaction mechanism. J. Photochem. Photobiol. A Chem. 2008, 198, 34-44.

15. Bouas-Laurent, H.; Castellan, A.; Desvergnea, J.-P.; Lapouyadec, R. Photodimerization of anthracenes in fluid solutions: (part 2) mechanistic aspects of the photocycloaddition and of the photochemical and thermal cleavage. Chem. Soc. Rev. 2001, 30, 248-263.

16. Schütz, A.; Wolff, T.J. Regioselectivity in the photodimerization of 9-hydroxy-methylanthracene and 9-anthracene carboxylic acid esters in surfactant systems. Photochem. Photobiol. A Chem. 1997, 109, 251-258.

17. Menon, S.; Thekkayil, R.; Varghese, S.; Das, S. Photoresponsive Soft Materials: Synthesis and Photophysical Studies of a Stilbene-Based Diblock Copolymer. J. Polym. Sci. A Polym. Chem. 2011, 49, 5063-5073.

18. Atta, A.M.; El-Ghazawy, R.A.; Farag, R.K.; El-Kafrawy, A.F.; Abdel-Azim, A.A. Crosslinked cinnamoyloxyethyl methacrylate and isooctyl acrylate copolymers as oil sorbers. Polym. Int. 2005, 54, 1088-1096.

19. Lendlein, A.; Jiang, H.; Jünger, O.; Langer, R. Light-induced shape-memory polymers. Nature 2005, 434, 879-882.

20. Tominaga, M.; Konishi, K.; Aida, T. A photocrosslinkable dendrimer consisting of a nucleobase. Chem. Lett. 2000, 29, 374-375.

21. Jiang, J.; Qi, B.; Lepage, M.; Zhao, Y. Polymer micelles stabilization on demand through reversible photo-cross-linking. Macromolecules 2007, 40, 790-792.

22. Boschmann, D.; Vana, P. Z-RAFT star polymerizations of acrylates: Star coupling via intermolecular chain transfer to polymer. Macromolecules 2007, 40, 2683-2693.

23. Boschmann, D.; Drache, M.; Fröhlich, M.; Zifferer, G.; Vana, P. Mechanism of Z-RAFT Star Polymerization. Polym. Prepr. 2008, 49, 189-190.

24. Boschmann, D.; Vana, P. Poly(vinyl acetate) and poly(vinyl propionate) star polymers via reversible addition fragmentation chain transfer (RAFT) polymerization. Polym. Bull. 2005, 53, 231-242. 
25. Gupta, S.; Kuckling, D.; Kretschmer, K.; Choudhary, V.; Adler, H.-J. Synthesis and characterization of stimuli-sensitive micro- and nanohydrogels based on photocrosslinkable poly(dimethylaminoethyl methacrylate). J. Polym. Sci. A Polym. Chem. 2007, 45, 669-679.

26. Neisen, B.; Steglich, W. Einfaches Verfahren zur Veresterung von Carbonsäuren. Angew. Chem. 1978, 90, 556-557.

27. Buback, M.; Junkers, T.; Vana, P. Laser single pulse initiated RAFT polymerization for assessing chain-length dependent radical termination kinetics. Macromol. Rapid Commun. 2005, 26, 796-802.

28. Beuermann, S.; Paquet, D.A.; McMinn, J.H.; Hutchinson, R.A. Determination of free-radical propagation rate coefficients of butyl, 2-ethylhexyl, and dodecyl acrylates by pulsed-laser polymerization. Macromolecules 1996, 29, 4206-4215.

29. Polymer Standard Service GmbH, 2012. Available online: http://www.polymer.de/ fileadmin/custom_documents/Gray-GPCSoftwareValidation-SympSanDiego96_01.pdf (accessed on 6 June 2013).

30. McKee, J.R.; Ladmiral, V.; Niskanen, J.; Tenhu, H.; Armes, S.P. Synthesis of Sterically-Stabilized Polystyrene Latexes Using Well-Defined Thermoresponsive Poly( $N$-isopropylacrylamide) Macromonomers. Macromolecules 2011, 44, 7692-7703.

31. Fröhlich, M.G.; Nardai, M.M.; Förster, N.; Vana, P.; Zifferer, G. Shielding effects in polymer-polymer reactions, 3. Z-RAFT Star Polymerization under Various Solvent Conditions. Polymer 2010, 51, 5122-5134.

32. Matsumoto, A.; Sano, Y.; Yoshioka, M.; Otsu, T. Kinetic study of radical polymerization of dialkyl fumarates using electron spin resonance spectroscopy. J. Polym. Sci. A Polym. Chem. 1996, 34, 291-299.

33. Lutz, J.-F.; Schmidt, B.V.K.J.; Pfeifer, S. Tailored Polymer Microstructures Prepared by Atom Transfer Radical Copolymerization of Styrene and N-substituted Maleimides. Macromol. Rapid Commun. 2011, 32, 127-135.

34. Kohli, P.; Scranton, A.B.; Blanchard, G.J. Co-polymerization of maleimides and vinyl ethers: A structural study. Macromolecules 1998, 31, 5681-5689.

35. Ganachaud, F.; Monteiro, M.J.; Gilbert, R.G. Pulsed-laser polymerization (PLP) of $N$-isopropyl acrylamide (NIPAM) in water: a qualitative study. Macromol. Symp. 2000, 150, 275-281.

36. Ganachaud, F.; Balic, R.; Monteiro, M.J.; Gilbert, R.G. Propagation rate coefficient of poly $(N$-isopropylacrylamide) in water below its lower critical solution temperature. Macromolecules 2000, 33, 8589-8596.

37. Asua, J.M.; Beuermann, S.; Buback, M.; Castignolles, P.; Charleux, B.; Gilbert, R.G.; Hutchinson, R.A.; Leiza, J.R.; Nikitin, A.N.; Vairon, J.P.; van Herk, A.M. Critically evaluated rate coefficients for free-radical polymerization, 5-Propagation rate coefficient for butyl acrylate. Macromol. Chem. Phys. 2004, 205, 2151-2160.

38. Ebeling, B.; Vana, P. Multiblock Copolymers of Styrene and Butyl Acrylate via Polytrithiocarbonate-Mediated RAFT Polymerization. Polymers 2011, 3, 719-739.

39. Kahne, D.; Still, W.C. Hydrolysis of a Peptide-Bond in Neutral Water. J. Am. Chem. Soc. 1988, 110, 7529-7534. 
40. Sinkel, C.; Greiner, A.; Agarwal, S. A Polymeric Drug Depot Based on 7-(2'-Methacryloyloxyethoxy)-4-methylcoumarin Copolymers for Photoinduced Release of 5-Fluorouracil Designed for the Treatment of Secondary Cataracts. Macromol. Chem. Phys. 2010, 211, 1857-1867.

41. Pregosin, P.S. NMR in Organometallic Chemistry; Wiley-VCH: Weinheim, Germany, 2012.

(C) 2013 by the authors; licensee MDPI, Basel, Switzerland. This article is an open access article distributed under the terms and conditions of the Creative Commons Attribution license (http://creativecommons.org/licenses/by/3.0/). 